\title{
Analysis of Three-Dimensional Fracture Mechanics Problems: A Two-Scale Approach Using Coarse Generalized FEM Meshes
}

\author{
D.-J. Kim, J.P.A. Pereira and C.A. Duarte \\ Department of Civil and Environmental Engineering, University of Illinois at Urbana-Champaign \\ 2122 Newmark Laboratory, 205 North Mathews Avenue \\ Urbana, Illinois 61801, USA
}

\begin{abstract}
SUMMARY
This paper presents a Generalized Finite Element Method (GFEM) based on the solution of interdependent global (structural) and local (crack) scale problems. The local problems focus on the resolution of fine-scale features of the solution in the vicinity of 3-D cracks while the global problem addresses the macro-scale structural behavior. The local solutions are embedded into the solution space for the global problem using the partition of unity method. The local problems are accurately solved using an $h p$-GFEM and thus the proposed method does not rely on analytical solutions. The proposed methodology enables accurate modeling of 3-D cracks on meshes with elements that are orders of magnitude larger than the process zone along crack fronts. The boundary conditions for the local problems are provided by the coarse global mesh solution and can be of Dirichlet, Neumann or Cauchy type. The effect of the type of local boundary condition on the performance of the proposed GFEM is analyzed. Several three-dimensional fracture mechanics problems aimed at investigating the accuracy of the method and its computational performance, both in terms of problem size and CPU time are presented.
\end{abstract}

KEY WORDS: Generalized FEM; Extended FEM; Fracture; Multiscale; Small cracks; Global-Local analysis.

\section{Introduction}

The Generalized or Extended FEM (G/XFEM) [3, 4, 8, 19, 50, 52, 63, 66] has been successfully applied to the simulation of boundary layers [16], propagating fractures [20, 35, 51, 67], acoustic problems [6, 48], polycrystalline microstructures [1, 62], etc. All of these applications have relied on closed-form enrichment functions that are known to approximate well the physics of the problem. However, analytical enrichment functions are in general not able to deliver accurate solutions on coarse three-dimensional meshes. To overcome this limitation, local mesh refinement must be performed as in the standard FEM [25, 57]. This creates several of the drawbacks of the FEM with remeshing and offsets many of the advantages of the G/XFEM. In the case of crack propagation and multiple site

\footnotetext{
${ }^{1}$ Correspondence to: C.A. Duarte, Department of Civil and Environmental Eng., University of Illinois at Urbana-Champaign, Newmark Laboratory, 205 North Mathews Avenue, Urbana, Illinois 61801, USA. Tel.: +1-217-244-2830; Fax: +1-217-3333821. e-mail: caduarte@illinois.edu.
}

* Submitted to International Journal of Numerical Methods in Engineering (March 13, 2009) 
damage analysis [2], the problem must be solved from scratch after each crack propagation step or for each crack configuration. Furthermore, the analysis of non-linear or time-dependent fracture problems may require mapping of solutions between meshes, as in the standard FEM. This may lead to loss of solution accuracy when the solution spaces are not nested. Even when analytical enrichments are able to approximate well the solution, as is the case in many 2-D fracture mechanics problems, the minimum crack size which can be modeled is controlled by the element size in the mesh [7].

In $[17,18,21,40]$ we demonstrate that accurate fracture mechanics solutions can be obtained in coarse meshes enriched with the so-called global-local enrichment functions. These functions are the solution of local boundary value problems defined in the neighborhood of cracks. Boundary conditions for these problems are provided by the coarse-scale global solution. We denote this class of methods as a GFEM with global-local enrichment functions $\left(G F E M^{\mathrm{gl}}\right)$. Global-local enrichment functions also enable the analysis of problems with sharp thermal gradients using coarse meshes, as demonstrated in [53].

In this paper, we combine the concept of global-local enrichments with the $h p$-GFEM for the 3D fractures presented in $[57,58]$. As a result, local features like cracks need not be discretized in global scale meshes. They are instead modeled by the solution of local problems. In addition, cracks that are smaller than global mesh elements can be discretized using this method. In this paper, we consider three types of boundary conditions applied to local problems: Dirichlet, Neumann and Cauchy. The effect of the type of local boundary condition on the performance of the proposed GFEM is analyzed.

From the approximation theory point of view, the proposed method is based on a two-scale decomposition of the solution-A smooth coarse-scale and a singular fine-scale component. The smooth component is approximated by a coarse global discretization of the domain. The fine-scale is locally approximated by the $h p-G F E M$ proposed in $[57,58]$. The partition of unity concept is used to paste the local approximations in the global solution space while still rendering a $C^{0}$ solution space. Details are presented in Section 3. Numerical examples demonstrate that the proposed method provides a two-way information transfer between coarse (structural) and fine (crack) scales while not requiring mesh refinement in structural scale meshes We also demonstrate that the method does not require the solution of the problem from scratch when analyzing several crack configurations in a mechanical component. This, as shown in Section 4, leads to a very efficient method for the class of problems considered here.

Several other two- or multi-scale approaches for the analysis of fracture mechanics problems have been proposed in recent years. A key difference among the various methods lies in the approach used to combine fine- and coarse-scale approximations, i.e., how to transfer information among scales. Among the recent works, we can mention the method of Guidault et al. [36] based on the LATIN method and domain decomposition concepts; the multigrid method proposed in [61]; the method of Cloirec et al. [13] based on Lagrange multipliers; the multiscale projection method of Belytschko et al. [10, 44]; the concurrent multiscale approach of Liu et al. [43, 46, 47]; the $h p$ FEM method of Krause et al. [27, 41]; the concurrent multi-level method of Gosh et at. [33, 34] based on the Voronoi Cell Finite Element Method; the multi-resolution approach proposed by Tsukanov and Shapiro [70] based on distance fields. The proposed $G F E M^{\mathrm{gl}}$ is also related to the refined global-local FEM proposed by Mao and Sun [45] and based on linear combinations of global and local approximations. The main difference with respect to the proposed $G F E M^{\mathrm{gl}}$ is, again, how the fine- and coarse-scale approximations are combined.

The s-version of the FEM (s-method) proposed by Fish et al. [30-32], the overlay technique of Belytschko et al. [9] and the combination of the s-method with the XFEM proposed by Lee et al. [42] can also be used to solve the class of problems considered in this paper. The s-method consists of overlaying a coarse finite element mesh with patches of fine meshes in regions where the solution 
exhibits high gradients or singularities [30]. A recent version of the s-method aimed at multiscale failure simulations, is the reduced order s-method (rs-method) of Fish et al. [28, 54]. Further discussion of some of these methods and their relations with the proposed $G F E M^{\mathrm{gl}}$ are presented in Sections 3.3 and 3.4.

Following this introduction, Section 2 presents a short summary of the Generalized Finite Element Method. Details on the proposed $G F E M^{\mathrm{gl}}$ are presented in Section 3. Section 4 presents several threedimensional fracture mechanics problems aimed at investigating the accuracy of the GFEM ${ }^{\mathrm{gl}}$ and its computational performance both in terms of problem size and CPU time.

\section{Generalized FEM: A Summary}

The generalized FEM $[3,4,19,52,63]$ is an instance of the so-called partition of unity method which has its origins in the works of Babuška et al. [4, 5, 49] and Duarte and Oden [15, 22-24, 52]. The extended FEM $[8,50]$ and several other methods proposed in recent years can also be formulated as special cases of the partition of unity method. In these methods, discretization spaces for a Galerkin method are defined using the concept of a partition of unity and approximation spaces that are selected based on a priori knowledge about the solution of a problem. A shape function, $\phi_{\alpha i}$, in the GFEM is computed from the product of a linear finite element shape function, $\varphi_{\alpha}$, and an enrichment function, $L_{\alpha i}$,

$$
\phi_{\alpha i}(\boldsymbol{x})=\varphi_{\alpha}(\boldsymbol{x}) L_{\alpha i}(\boldsymbol{x}) \quad(\text { no summation on } \alpha)
$$

where $\alpha$ is a node in the finite element mesh. Figure 1 illustrates the construction of GFEM shape functions.

The Lagrangian finite element shape functions $\varphi_{\alpha}, \alpha=1, \ldots, N$, in a finite element mesh with $N$ nodes constitute a partition of unity, i.e., $\sum_{\alpha=1}^{N} \varphi_{\alpha}(x)=1$ for all $x$ in a domain $\Omega$ covered by the finite element mesh. This is a key property used in partition of unity methods. Linear combinations of the GFEM shape functions $\phi_{\alpha i}, \alpha=1, \ldots, N$, can represent exactly any enrichment function $L_{\alpha i}[15,23]$.

Several enrichment functions can be hierarchically added to any node $\alpha$ in a finite element mesh. Thus, if $D_{L}$ is the number of enrichment functions at node $\alpha$, the GFEM approximation, $\boldsymbol{u}^{h p}$, of a vector field $\boldsymbol{u}$ can be written as

$$
\begin{aligned}
\boldsymbol{u}^{h p}(\boldsymbol{x}) & =\sum_{\alpha=1}^{N} \sum_{i=1}^{D_{L}} \underline{\boldsymbol{u}}_{\alpha i} \phi_{\alpha i}(\boldsymbol{x})=\sum_{\alpha=1}^{N} \sum_{i=1}^{D_{L}} \underline{\boldsymbol{u}}_{\alpha i} \varphi_{\alpha}(\boldsymbol{x}) L_{\alpha i}(\boldsymbol{x}) \\
& =\sum_{\alpha=1}^{N} \varphi_{\alpha}(\boldsymbol{x}) \sum_{i=1}^{D_{L}} \underline{\boldsymbol{u}}_{\alpha i} L_{\alpha i}(\boldsymbol{x})=\sum_{\alpha=1}^{N} \boldsymbol{\varphi}_{\alpha}(\boldsymbol{x}) \boldsymbol{u}_{\alpha}^{h p}(\boldsymbol{x})
\end{aligned}
$$

where $\underline{\boldsymbol{u}}_{\alpha i}, \alpha=1, \ldots, N, i=1, \ldots, D_{L}$, are nodal degrees of freedom and $\boldsymbol{u}_{\alpha}^{h p}(\boldsymbol{x})$ is an approximation of $\boldsymbol{u}$ defined on $\omega_{\alpha}=\left\{\boldsymbol{x} \in \Omega: \varphi_{\alpha}(\boldsymbol{x}) \neq 0\right\}$, the support of the partition of unity function $\varphi_{\alpha}$. In the case of a finite element partition of unity, the support $\omega_{\alpha}$ (often called cloud) is given by the union of the finite elements sharing a vertex node $\boldsymbol{x}_{\alpha}$ [19]. The equation above shows that the global GFEM approximation $\boldsymbol{u}^{h p}(\boldsymbol{x})$ is built by pasting together cloud-wise approximations $\boldsymbol{u}_{\alpha}^{h p}, \alpha=1, \ldots, N$, using a partition of unity.

The cloud approximations $\boldsymbol{u}_{\alpha}^{h p}, \alpha=1, \ldots, N$, belong to spaces $\chi_{\alpha}\left(\omega_{\alpha}\right)=\operatorname{span}\left\{L_{i \alpha}\right\}_{i=1}^{D_{L}}$ defined on the supports $\omega_{\alpha}, \alpha=1, \ldots, N$. A-priori knowledge about the behavior of the function $\boldsymbol{u}$ over the cloud $\omega_{\alpha}$ is used when selecting enrichment or basis functions for a particular space $\chi_{\alpha}\left(\omega_{\alpha}\right)$. We refer to 


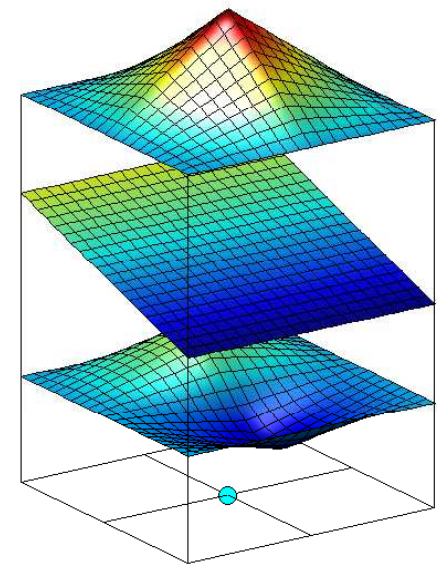

(a)

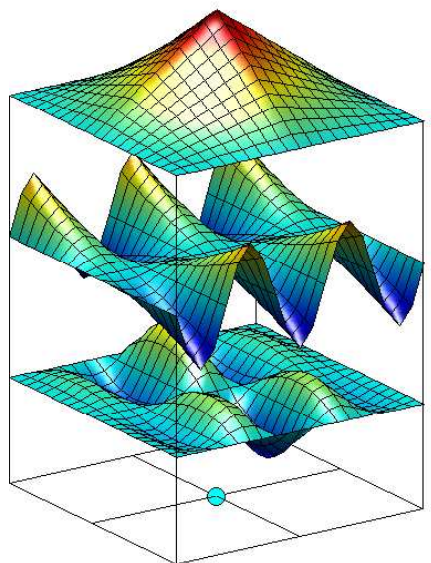

(b)

Figure 1: Construction of a generalized FEM shape function using a polynomial (a) and a non-polynomial enrichment (b). Here, $\varphi_{\alpha}$ are the functions at the top, the enrichment functions, $L_{\alpha i}$, are the functions in the middle, and the generalized FE shape functions, $\phi_{\alpha i}$, are the resulting bottom functions.

$[12,19,20,35,51,57,58,66,67]$ and the references therein, for details on the selection of these functions for the case 3-D linear elastic fracture mechanics problems like those considered in this paper.

In $[57,58]$, we show that available analytical enrichments for 3-D fracture problems enable modeling of surface discontinuities arbitrarily located within a finite element mesh (across elements). Nonetheless, a sufficiently fine mesh must be used around the crack front to achieve acceptable accuracy. Even though the refinement does not have to be as strong as in the FEM, it still creates many of the problems faced by the FEM when simulating, for example, propagating cracks or performing a multi-site damage analysis. Mesh refinement around the cracks requires that the problem be solved from scratch for each crack configuration, leading to high computational costs.

\section{Solution of Two-Scale Problems Using Global-Local Enrichments}

In $[17,18,21]$ we present a procedure to build enrichment functions based on the solution of local boundary value problems defined in the neighborhood of cracks. The boundary conditions for these problems are provided by a GFEM solution computed on coarse global meshes. We denote this class of methods as a GFEM with global-local enrichment functions $\left(\right.$ GFEM $\left.^{\mathrm{gl}}\right)$. In $[17,18,21]$ cracks are discretized in the global meshes which prevents, for example, the analysis of small cracks or other fine-scale features while keeping the global mesh coarse. In this section, this limitation is removed through a two-scale decomposition of the solution of the global problem. The key idea is to combine the global-local procedure of the $G F E M^{\mathrm{gl}}$ with the $h p-G F E M$ presented in $[57,58]$. The latter is used to discretize the local boundary value problems used in the FFEM $^{\mathrm{gl}}$ and thus the proposed methodology enables modeling of small cracks on coarse, uncracked, global meshes. Details are presented next.

[GFEMgl'two`scale - July 1, 2009] 


\subsection{Formulation of Coarse-Scale Global Problem}

Consider a domain $\bar{\Omega}_{G}=\Omega_{G} \cup \partial \Omega_{G}$ in $\Re^{3}$. The boundary is decomposed as $\partial \Omega_{G}=\partial \Omega_{G}^{u} \cup \partial \Omega_{G}^{\sigma}$ with $\partial \Omega_{G}^{u} \cap \partial \Omega_{G}^{\sigma}=\emptyset$. The equilibrium equations are given by

$$
\nabla \cdot \boldsymbol{\sigma}=\mathbf{0} \quad \text { in } \Omega_{G}
$$

The constitutive relations are given by the generalized Hooke's law, $\boldsymbol{\sigma}=\boldsymbol{C}: \boldsymbol{\varepsilon}$, where $\boldsymbol{C}$ is Hooke's tensor. The following boundary conditions are prescribed on $\partial \Omega_{G}$

$$
\boldsymbol{u}=\overline{\boldsymbol{u}} \text { on } \partial \Omega_{G}^{u} \quad \boldsymbol{\sigma} \cdot \boldsymbol{n}=\overline{\boldsymbol{t}} \text { on } \partial \Omega_{G}^{\sigma},
$$

where $n$ is the outward unit normal vector to $\partial \Omega_{G}^{\sigma}$ and $\overline{\boldsymbol{t}}$ and $\overline{\boldsymbol{u}}$ are prescribed tractions and displacements, respectively.

Let $\boldsymbol{u}_{G}^{0}$ denote the generalized or standard FEM solution of the problem defined by (2), (3). This is hereafter denoted as the initial global problem. The approximation $\boldsymbol{u}_{G}^{0}$ is the solution of the following problem:

Find $\boldsymbol{u}_{G}^{0} \in \boldsymbol{X}_{G}^{0}\left(\Omega_{G}\right) \subset H^{1}\left(\Omega_{G}\right)$ such that, $\forall \boldsymbol{v}_{G}^{0} \in \boldsymbol{X}_{G}^{0}\left(\Omega_{G}\right)$

$$
\int_{\Omega_{G}} \boldsymbol{\sigma}\left(\boldsymbol{u}_{G}^{0}\right): \boldsymbol{\varepsilon}\left(\boldsymbol{v}_{G}^{0}\right) d \boldsymbol{x}+\eta \int_{\partial \Omega_{G}^{u}} \boldsymbol{u}_{G}^{0} \cdot \boldsymbol{v}_{G}^{0} d \boldsymbol{s}=\int_{\partial \Omega_{G}^{\sigma}} \overline{\boldsymbol{t}} \cdot \boldsymbol{v}_{G}^{0} d \boldsymbol{s}+\eta \int_{\partial \Omega_{G}^{u}} \overline{\boldsymbol{u}} \cdot \boldsymbol{v}_{G}^{0} d \boldsymbol{s}
$$

where, $\boldsymbol{X}_{G}^{0}\left(\Omega_{G}\right)$ is a discretization of $H^{1}\left(\Omega_{G}\right)$, a Hilbert space defined on $\Omega_{G}$, built with generalized, or standard, FEM shape functions. In this paper, the GFEM is used and the space $\boldsymbol{X}_{G}^{0}\left(\Omega_{G}\right)$ is given by

$$
\boldsymbol{X}_{G}^{0}\left(\Omega_{G}\right)=\left\{\boldsymbol{u}^{h p}=\sum_{\alpha=1}^{N} \varphi_{\alpha}(\boldsymbol{x}) \hat{\boldsymbol{u}}_{\alpha}^{h p}(\boldsymbol{x}): \hat{\boldsymbol{u}}_{\alpha}^{h p}(\boldsymbol{x})=\sum_{i=1}^{\hat{D}_{L}} \underline{\hat{\boldsymbol{u}}}_{\alpha i} \hat{L}_{\alpha i}(\boldsymbol{x})\right\}
$$

where $\underline{\hat{\boldsymbol{u}}}_{\alpha i}, \alpha=1, \ldots, N, i=1, \ldots, \hat{D}_{L}$, are nodal degrees of freedom and $\hat{D}_{L}$ is the dimension of a set of polynomial enrichment functions, $\hat{L}_{\alpha i}(\boldsymbol{x})$, of degree less than or equal to $p-1$. Details can be found, for example, in Section 3.2 of [57]. Space $\boldsymbol{X}_{G}^{0}\left(\Omega_{G}\right)$ can also be defined using standard polynomial FEM shape functions since cracks are not discretized in the initial global problem.

The parameter $\eta$ in (4) is a penalty parameter. We use the penalty method due to its simplicity and generality. Other methods to impose Dirichlet boundary conditions can be used as well.

The mesh used to solve problem (4) is typically a coarse quasi-uniform mesh like the one illustrated in Figure 2. This mesh and the solution $\boldsymbol{u}_{G}^{0}$ are usually available from the design phase of the structure or mechanical component.

\subsection{Formulation of Fine-Scale Problem}

The proposed approach involves the solution of a local boundary value problem defined in a neighborhood $\Omega_{L}$ of a crack and subjected to boundary conditions provided by the global solution $\boldsymbol{u}_{G}^{0}$ (Cf. Figure 2). In this paper, we generalize the formulation introduced in [17, 18, 21] by considering the cases of Dirichlet, Neumann and Cauchy boundary conditions provided by the global solution $\boldsymbol{u}_{G}^{0}$. In each case, a local problem is solved on $\Omega_{L}$ after the global solution $\boldsymbol{u}_{G}^{0}$ is computed as described above.

The statement of the principle of virtual work for the local problem is given by 


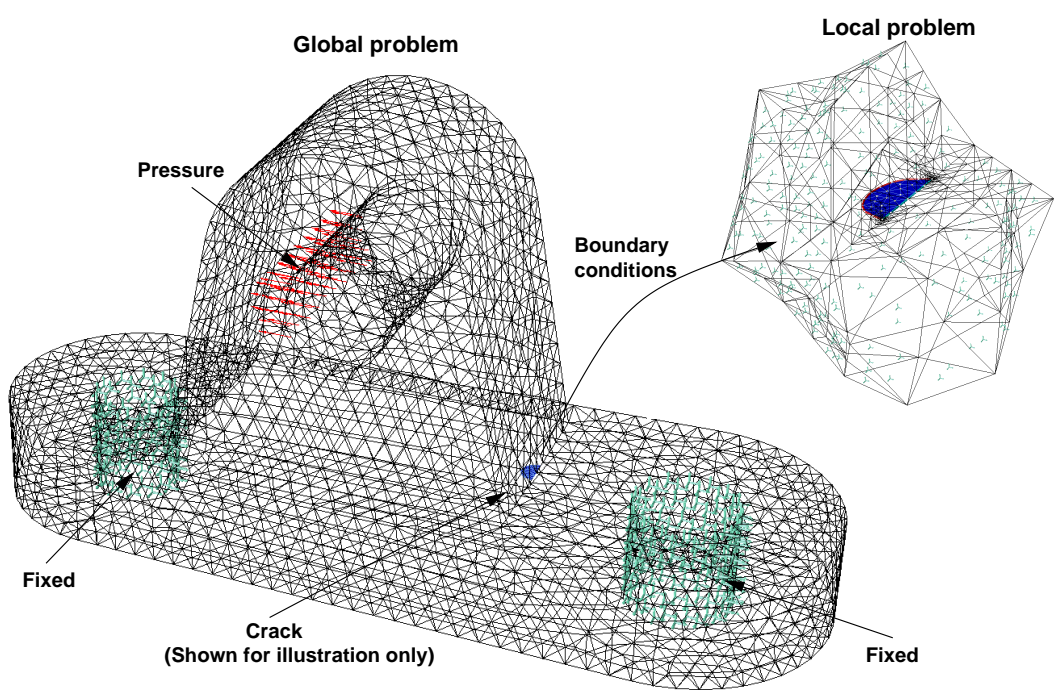

Figure 2: Model problem used to illustrate the main ideas of the $G F E M^{\mathrm{gl}}$. The figure shows a crack in a 3$\mathrm{D}$ bracket. The solution computed on the coarse global mesh provides boundary conditions for the extracted local domain in a neighborhood of the crack. The crack is shown in the global domain for illustration purposes only. In the proposed $G F E M^{\mathrm{gl}}$, fine-scale features are not discretized in the global problem. Instead, global-local enrichment functions are used.

Find $\boldsymbol{u}_{L} \in \boldsymbol{X}_{L}^{h p}\left(\Omega_{L}\right) \subset H^{1}\left(\Omega_{L}\right)$ such that, $\forall \boldsymbol{v}_{L} \in \boldsymbol{X}_{L}^{h p}\left(\Omega_{L}\right)$

$$
\begin{aligned}
& \int_{\Omega_{L}} \boldsymbol{\sigma}\left(\boldsymbol{u}_{L}\right): \boldsymbol{\varepsilon}\left(\boldsymbol{v}_{L}\right) d \boldsymbol{x}+\eta \int_{\partial \Omega_{L} \cap \partial \Omega_{G}^{u}} \boldsymbol{u}_{L} \cdot \boldsymbol{v}_{L} d \boldsymbol{s}+\kappa \int_{\partial \Omega_{L} \backslash\left(\partial \Omega_{L} \cap \partial \Omega_{G}\right)} \boldsymbol{u}_{L} \cdot \boldsymbol{v}_{L} d \boldsymbol{s}= \\
& \int_{\partial \Omega_{L} \cap \partial \Omega_{G}^{\sigma}} \overline{\boldsymbol{t}} \cdot \boldsymbol{v}_{L} d \boldsymbol{s}+\eta \int_{\partial \Omega_{L} \cap \partial \Omega_{G}^{u}} \overline{\boldsymbol{u}} \cdot \boldsymbol{v}_{L} d \boldsymbol{s}+\int_{\partial \Omega_{L} \backslash\left(\partial \Omega_{L} \cap \partial \Omega_{G}\right)}\left(\boldsymbol{t}\left(\boldsymbol{u}_{G}^{0}\right)+\kappa \boldsymbol{u}_{G}^{0}\right) \cdot \boldsymbol{v}_{L} d \boldsymbol{s}
\end{aligned}
$$

where $\boldsymbol{X}_{L}^{h p}\left(\Omega_{L}\right)$ is a discretization of $H^{1}\left(\Omega_{L}\right)$ using the GFEM shape functions presented in [57, 58]

$$
\boldsymbol{X}_{L}^{h p}\left(\Omega_{L}\right)=\left\{\boldsymbol{u}^{h p}=\sum_{\alpha=1}^{N_{L}} \boldsymbol{\varphi}_{\alpha}(\boldsymbol{x})\left[\hat{\boldsymbol{u}}_{\alpha}^{h p}(\boldsymbol{x})+\mathscr{H} \tilde{\boldsymbol{u}}_{\alpha}^{h p}(\boldsymbol{x})+\breve{\boldsymbol{u}}_{\alpha}^{h p}(\boldsymbol{x})\right]\right\}
$$

The partition of unity functions, $\varphi_{\alpha}, \alpha=1, \ldots, N_{L}$, are linear Lagrangian shape functions defined by a finite element discretization of $\Omega_{L}$. The summation limit, $N_{L}$, is the number of nodes in this mesh. The cloud-wise functions $\hat{\boldsymbol{u}}_{\alpha}^{h p}(\boldsymbol{x}), \mathscr{H} \tilde{\boldsymbol{u}}_{\alpha}^{h p}(\boldsymbol{x})$ and $\breve{\boldsymbol{u}}_{\alpha}^{h p}(\boldsymbol{x})$ are approximations of the continuous, discontinuous and singular components of the solution, respectively. The mesh used in $\Omega_{L}$ does not fit the crack surface. The crack is modeled instead by these functions. Details can be found in Section 3.2 of [57].

The traction vector, $\boldsymbol{t}\left(\boldsymbol{u}_{G}^{0}\right)$, that appears in the integral over $\partial \Omega_{L} \backslash\left(\partial \Omega_{L} \cap \partial \Omega_{G}\right)$ is computed from the coarse-scale solution using Cauchy's relation, i.e.,

$$
\boldsymbol{t}\left(\boldsymbol{u}_{G}^{0}\right)=\hat{\boldsymbol{n}} \cdot \boldsymbol{\sigma}\left(\boldsymbol{u}_{G}^{0}\right)=\hat{\boldsymbol{n}} \cdot\left(\boldsymbol{C}: \boldsymbol{\varepsilon}\left(\boldsymbol{u}_{G}^{0}\right)\right)
$$

[GFEMgl'two`scale - July 1, 2009] 
with $\hat{\boldsymbol{n}}$ the outward unit normal vector to $\partial \Omega_{L}$. The parameters $\eta$ and $\kappa$ are a penalty parameter and a spring stiffness defined on $\partial \Omega_{L} \cap \partial \Omega_{G}^{u}$ and $\partial \Omega_{L} \backslash\left(\partial \Omega_{L} \cap \partial \Omega_{G}\right)$, respectively.

We can select the type of boundary conditions provided by $\boldsymbol{u}_{G}^{0}$ depending on the choice of spring stiffness $\kappa$ as follows:

(i) Neumann boundary condition: Set $\kappa=0$. Tractions defined in (8) are prescribed on $\partial \Omega_{L} \backslash\left(\partial \Omega_{L} \cap\right.$ $\left.\partial \Omega_{G}\right)$.

Note that problem (6) may be not well-posed if $\partial \Omega_{L} \cap \partial \Omega_{G}^{u}=\emptyset$, since, in this case, it is a pure Neumann problem. The tractions applied on $\partial \Omega_{L}$ are in general not equilibrated since they are computed from the coarse-scale GFEM solution. However, when solving simple uncracked global domains subjected to uniaxial loads like in the problem of Section 4.2, the coarsescale solution $\boldsymbol{u}_{G}^{0}$ is exact. Thus, the local Neumann problems are well-posed ${ }^{1}$. An example is presented in Section 4.2.

(ii) Dirichlet boundary condition: Set $\kappa=\eta \gg 1$. In this case, the solution $\boldsymbol{u}_{G}^{0}$ of the initial global problem is used as Dirichlet boundary condition on $\partial \Omega_{L} \backslash\left(\partial \Omega_{L} \cap \partial \Omega_{G}\right)$. The performance of this choice of boundary condition is analyzed in [18].

(iii) Cauchy or spring boundary condition: Set $0<\kappa<\eta$. Cauchy boundary conditions are given by [68]

$$
\boldsymbol{t}(\boldsymbol{u})=\kappa(\boldsymbol{\delta}-\boldsymbol{u})
$$

where $\boldsymbol{t}$ is the prescribed traction, $\kappa$ is the stiffness of the springs, $\boldsymbol{\delta}$ is displacement imposed at the base of the spring system and $\boldsymbol{u}$ is the displacement at the boundary of the body [68]. From the above we have that

$$
\kappa \boldsymbol{\delta}=\boldsymbol{t}+\kappa \boldsymbol{u}
$$

Since $\boldsymbol{t}$ and $\boldsymbol{u}$ are not known, we use instead values provided by the coarse-scale solution $\boldsymbol{u}_{G}^{0}$ and set

$$
\kappa \boldsymbol{\delta}:=\boldsymbol{t}\left(\boldsymbol{u}_{G}^{0}\right)+\kappa \boldsymbol{u}_{G}^{0}
$$

With this choice, the tractions on $\partial \Omega_{L} \backslash\left(\partial \Omega_{L} \cap \partial \Omega_{G}\right)$ are given by

$$
\boldsymbol{t}(\boldsymbol{u})=\boldsymbol{t}\left(\boldsymbol{u}_{G}^{0}\right)+\kappa \boldsymbol{u}_{G}^{0}-\kappa \boldsymbol{u}
$$

Thus, the prescribed tractions will be close to the case of Neumann boundary condition discussed above if $\boldsymbol{u}_{G}^{0}$ is close to $\boldsymbol{u}$. However, in this case, the local problem is well-posed even if the tractions $\boldsymbol{t}\left(\boldsymbol{u}_{G}^{0}\right)$ are not equilibrated.

There is a great freedom in selecting the spring constant $\kappa$ as shown in Section 4 . If $\kappa$ is taken as a large value (compared with the stiffness of the body), the boundary condition degenerates to a Dirichlet boundary condition. Our numerical experiments show that any value of $\kappa$ comparable to, or larger than the stiffness of the body is acceptable and provides global-local enrichment functions with good approximation properties.

\footnotetext{
${ }^{1}$ Even when $\boldsymbol{u}_{G}^{0}$ is the exact solution of (4), a Neumann local problem may be not equilibrated due to roundoff or integration errors [11]. Our main goal in considering the case $\kappa=0$ is to compare the performance of different types of boundary conditions prescribed on $\partial \Omega_{L} \backslash\left(\partial \Omega_{L} \cap \partial \Omega_{G}\right)$.

[GFEMgl'two`scale - July 1, 2009]
} 


\subsection{Global-Local Enrichment Functions and Enriched Global Problem}

The solution $\boldsymbol{u}_{L}$ of the local problem defined above can be used to build generalized FEM shape functions for the coarse global mesh. Equation (1) is used with the partition of unity function, $\varphi_{\alpha}$, provided by the global, coarse, FE mesh and the enrichment function given by $\boldsymbol{u}_{L}$, i.e.,

$$
\phi_{\alpha}(x)=\varphi_{\alpha}(x) u_{L}(x)
$$

Hereafter, $\boldsymbol{u}_{L}$ is denoted a global-local enrichment function and the global problem enriched with these functions is denoted an enriched global problem. The formulation of this problem is given by

Find $\boldsymbol{u}_{G}^{E} \in \boldsymbol{X}_{G}^{E}\left(\Omega_{G}\right) \subset H^{1}\left(\Omega_{G}\right)$ such that, $\forall \boldsymbol{v}_{G}^{E} \in \boldsymbol{X}_{G}^{E}\left(\Omega_{G}\right)$

$$
\int_{\Omega_{G}} \boldsymbol{\sigma}\left(\boldsymbol{u}_{G}^{E}\right): \boldsymbol{\varepsilon}\left(\boldsymbol{v}_{G}^{E}\right) d \boldsymbol{x}+\eta \int_{\partial \Omega_{G}^{u}} \boldsymbol{u}_{G}^{E} \cdot \boldsymbol{v}_{G}^{E} d \boldsymbol{s}=\int_{\partial \Omega_{G}^{\boldsymbol{\sigma}}} \overline{\boldsymbol{t}} \cdot \boldsymbol{v}_{G}^{E} d \boldsymbol{s}+\eta \int_{\partial \Omega_{G}^{u}} \overline{\boldsymbol{u}} \cdot \boldsymbol{v}_{G}^{E} d \boldsymbol{s}
$$

where, $\boldsymbol{X}_{G}^{E}\left(\Omega_{G}\right)$ is the space $\boldsymbol{X}_{G}^{0}\left(\Omega_{G}\right)$ augmented with GFEM functions (10), i.e.,

$$
\boldsymbol{X}_{G}^{E}\left(\Omega_{G}\right)=\{\boldsymbol{u}^{h p}=\underbrace{\sum_{\alpha=1}^{N} \varphi_{\alpha}(\boldsymbol{x}) \hat{\boldsymbol{u}}_{\alpha}^{h p}(\boldsymbol{x})}_{\text {coarse-scale approx. }}+\underbrace{\sum_{\beta \in \mathscr{I}_{g l}} \varphi_{\beta}(\boldsymbol{x}) \boldsymbol{u}_{\beta}^{g l}(\boldsymbol{x})}_{\text {fine-scale approx. }}\}
$$

where $\mathscr{I}_{g l}$ is the index set of nodes enriched with function $\boldsymbol{u}_{L}, \hat{\boldsymbol{u}}_{\alpha}^{h p}$ is defined in (5) and

$$
\boldsymbol{u}_{\beta}^{g l}(\boldsymbol{x})=\left[\begin{array}{ll}
\underline{u}_{\beta 1} & u_{L 1}(\boldsymbol{x}) \\
\underline{u}_{\beta 2} & u_{L 2}(\boldsymbol{x}) \\
\underline{u}_{\beta 3} & u_{L 3}(\boldsymbol{x})
\end{array}\right]
$$

where $\underline{u}_{\beta j}, \beta \in \mathscr{I}_{g l}, j=1,2,3$, are nodal degrees of freedom and $u_{L j}(\boldsymbol{x}), j=1,2,3$, are Cartesian components of displacement vector $\boldsymbol{u}_{L}$. The coarse-scale approximation may also include the cloudwise discontinuous functions $\mathscr{H} \tilde{\boldsymbol{u}}_{\alpha}^{h p}(\boldsymbol{x})$ discussed in Section 3.2. These functions are hierarchically added to the global solution space if the local domain $\Omega_{L}$ does not contain the entire crack surface, as in the example of Section 4.2.

The enriched global problem is solved on the same coarse global mesh used in the computation of the initial global problem (4). Global-local enrichments add only three degrees of freedom to each node $\beta \in \mathscr{I}_{g l}$ of the global mesh when solving a 3-D elasticity problem, regardless of the number of degrees of freedom of the local problem (several thousands in general). Thus, highly adapted local discretizations able to capture fine-scale features of the solution can be used at the local problem, since the level of local mesh refinement/enrichment does not impact the size of the global problem. This contrasts with the FEM, which requires very fine global discretizations in order to capture small-scale behavior in the global domain. Figure 3 illustrates the enrichment of the global coarse mesh with the solution of a local problem defined in a neighborhood of a crack.

As mentioned in Section 3.1, the coarse-scale global problem can be solved using the standard FEM since no cracks or fine-scale features are modeled in that problem. In this case, the enriched global 


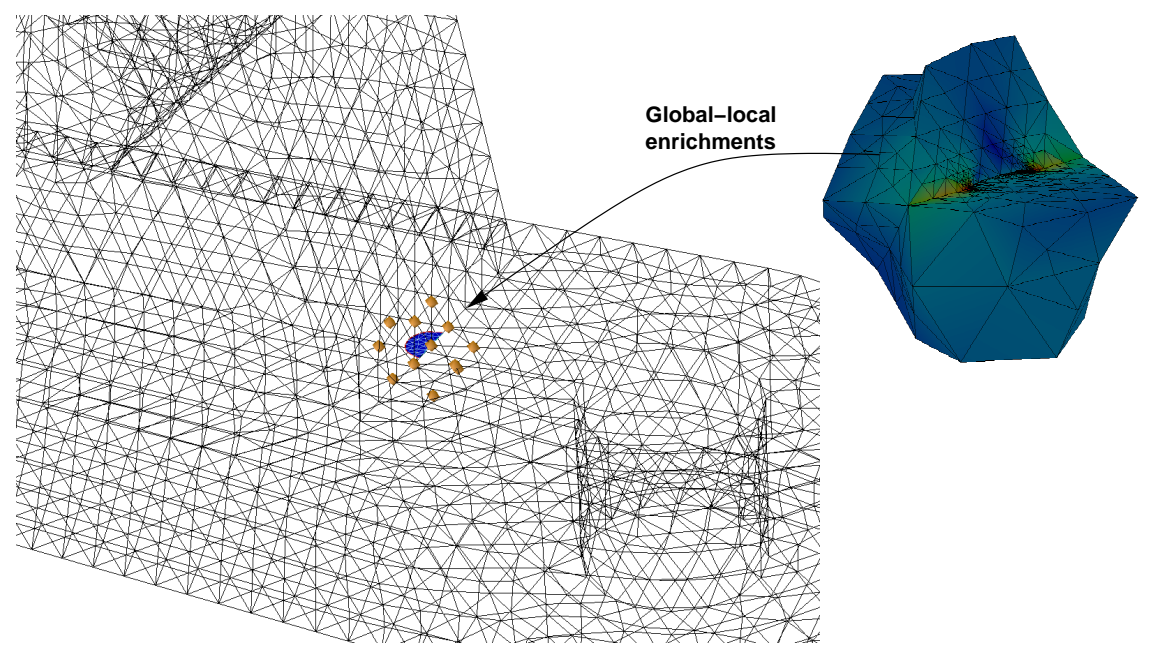

Figure 3: Enrichment of the coarse global mesh with a local solution. Only three degrees of freedom are added to nodes with yellow glyphs. The crack is shown in the global domain for illustration purposes only.

space $\boldsymbol{X}_{G}^{E}\left(\Omega_{G}\right)$ is given by

$$
\boldsymbol{X}_{G}^{E}\left(\Omega_{G}\right)=\{\boldsymbol{u}^{h p}=\underbrace{\sum_{\alpha=1}^{N} \varphi_{\alpha}(\boldsymbol{x}) \underline{\hat{\boldsymbol{u}}}_{\alpha}}_{\text {standard FEM approx. }}+\underbrace{\sum_{\beta \in \mathscr{I}_{g l}} \varphi_{\beta}(\boldsymbol{x}) \boldsymbol{u}_{\beta}^{g l}(\boldsymbol{x})}_{\text {fine-scale approx. }}\}
$$

where $\underline{\hat{\boldsymbol{u}}}_{\alpha}, \alpha=1, \ldots, N$, are (standard) nodal degrees of freedom. The finite element partition of unity functions, $\varphi_{\alpha}, \alpha=1, \ldots, N$, can be linear, quadratic or high-order Lagrangian shape functions. Thus, if a GFEM code is available for the computation of the global-local enrichment function $\boldsymbol{u}_{L}$, the proposed $G_{F E M}{ }^{\mathrm{gl}}$ can be implemented in existing FEM codes. The numerical integration of global-local shape functions (10) must, of course, be properly handled. This is discussed in Section 3.5.

Related Methods In addition to the various methods discussed in Section 1, the proposed $G F E M^{\mathrm{gl}}$ is also related to the so-called mesh-based handbook approach of Strouboulis et al. [63-65] and the upscaling technique proposed by Hou and Xu [39]. We refer the reader to Section 3.3 of reference [18] for a discussion on the relations among these methods.

\subsection{Solution of enriched global problem}

It is clear from the definition of the enriched global space $\boldsymbol{X}_{G}^{E}\left(\Omega_{G}\right)$ given in (12), that the globallocal GFEM shape functions are hierarchically added to the coarse-scale space $\boldsymbol{X}_{G}^{0}\left(\Omega_{G}\right)$. As a result, the global stiffness matrix of the initial global problem (4), $\boldsymbol{K}_{G}^{0}$, is nested in the global matrix of the enriched problem (11), $\boldsymbol{K}_{G}^{E}$. Matrix $\boldsymbol{K}_{G}^{E}$ can be partitioned as follows (see also Section A.2 of [18])

$$
\left[\begin{array}{ll}
\boldsymbol{K}_{G}^{0} & \boldsymbol{K}_{G}^{0, g l} \\
\boldsymbol{K}_{G}^{\mathrm{gl}, 0} & \boldsymbol{K}_{G}^{\mathrm{gl}}
\end{array}\right]\left[\begin{array}{l}
\tilde{u}_{G}^{0} \\
\underline{\underline{u}}_{G}^{\mathrm{gl}}
\end{array}\right]=\left[\begin{array}{l}
\boldsymbol{F}_{G}^{0} \\
\boldsymbol{F}_{G}^{\mathrm{gl}}
\end{array}\right]
$$


where $\boldsymbol{K}_{G}^{\mathrm{gl}}$ and $\underline{\boldsymbol{u}}_{G}^{\mathrm{gl}}$ are the global entries and degrees of freedom, respectively, associated with hierarchical global-local enrichments. Vector $\underline{u}_{G}^{\mathrm{gl}}$ contains the degrees of freedom $\underline{u}_{\beta j}, \beta \in \mathscr{I}_{g l}, j=$ $1,2,3$, defined in Section 3.3. As such, its dimension is small compared with that of vector $\underline{\tilde{u}}_{G}^{0}$. In the example of Section 4.3 (crack case 1 or 2$), \operatorname{dim}\left(\underline{\boldsymbol{u}}_{G}^{\mathrm{gl}}\right)=27$ while $\operatorname{dim}\left(\underline{\tilde{\boldsymbol{u}}}_{G}^{0}\right)=115,470$. This is in contrast with the s-method proposed in, e.g., [42] where the number of hierarchical degrees of freedom is equal to the dimension of the local problem. In the case of the example of Section 4.3 (crack case 1), the dimension of the local problem is equal to 23,268 .

The hierarchical nature and the small size of $\underline{u}_{G}^{\mathrm{gl}}$ can be explored to efficiently solve the enriched global system of equations (14). In this paper, the algorithm proposed in Section A.2 of [18] is employed. In this approach, the global-local degrees of freedom $\underline{u}_{\beta j}, \beta \in \mathscr{I}_{g l}, j=1,2,3$, are condensed out using the available factorization of the initial global problem. A similar approach is used by Hirai et al. [37, 38] in the framework of the global-local FEM [29]. Here, however, the number of degrees of freedom to be condensed out is much smaller than in the cases considered by Hirai et al. $[37,38]$. Other approaches that could be used to efficiently solve the enriched global problem include the iterative methods of Rank et al. [27, 41], Düster [26], and of Whitcomb [72].

Substructuring [29] can also be used to solve the class of problems considered in this paper and this approach was combined with the XFEM in [73, 74]. Like in the GFEM $^{\mathrm{gl}}$, the condensed substructure adds only a few degrees of freedom (dofs) to the global system of equations. However, those dofs are not hierarchical with respect to the global, uncracked, discretization. Therefore, the problem must, in general, be solved from scratch for each crack location/configuration. It has also been reported in the literature that substructuring may lead to ill-conditioned systems when the difference in element sizes in the global and local meshes is large [36].

\subsection{Numerical integration}

In the proposed $G F E M^{\mathrm{gl}}$, the elements enriched with global-local enrichment functions can be integrated efficiently and accurately. This is possible since the local meshes are nested in the global mesh. Figure 4 illustrates the numerical integration procedure adopted in this paper. It is basically a combination of the approaches proposed in Section A.3 of [18] and Section 4.2 of [57]. The orange line in the figure represents a crack surface cutting elements in the mesh. A yellow square indicates a node of the global mesh enriched with global-local enrichment functions. The numerical integration over global computational elements connected to these nodes is performed with the aid of local problem elements nested in the global elements. These elements are denoted as local computational elements. They are used to define quadrature points and weights as illustrated in Figure 4(b). Standard quadrature rules are used at local elements not cut by the crack surface or enriched with singular functions. Otherwise, the local elements are subdivided in the so-called integration elements as discussed, for example, in Section 4.2 of [57]. Special quadrature rules, such as those proposed in [55], may be used at local elements enriched with singular functions.

The implementation of the above scheme involves the following mappings:

- from master coordinates of a local computational element to master coordinates of a global computational element;

- from master coordinates of a local integration element to master coordinates of a global computational element;

- from master coordinates of a local integration element to master coordinates of a local computational element.

[GFEMgl'two'scale - July 1, 2009] 
The master coordinates in the first two cases are used in the computation of the global partition of unity $\varphi_{\alpha}$ in (10), while the third mapping is required to retrieve the local solution $\boldsymbol{u}_{L}$. In all cases, the mapping is performed by first computing the global physical coordinates $\boldsymbol{x}$ of an integration point in the original element followed by the mapping of $x$ to the master coordinates of a global or local computational element. No search of the element containing $\boldsymbol{x}$ is required thanks to the nesting of meshes as described above. The inverse mapping of $\boldsymbol{x}$ to the master coordinates of a global or local computational element can be done in a closed-form in the case of tetrahedral and triangular elements. Thus, the numerical overhead involved is small as demonstrated in [18].

The integration order of local computational or integration elements nested in global computational elements is taken as the maximum of the integration orders of its polynomial enrichment functions $\hat{L}_{\alpha i}$ in (5) and global-local enrichment functions $\boldsymbol{u}_{L}$ in (10) plus one. The integration order is increased by one since the global partition of unity is a linear finite element shape function. This strategy provides a systematic way of accurately and efficiently integrating GFEM shape functions with global-local enrichments. Further details on the procedure can be found in [18] and [57].

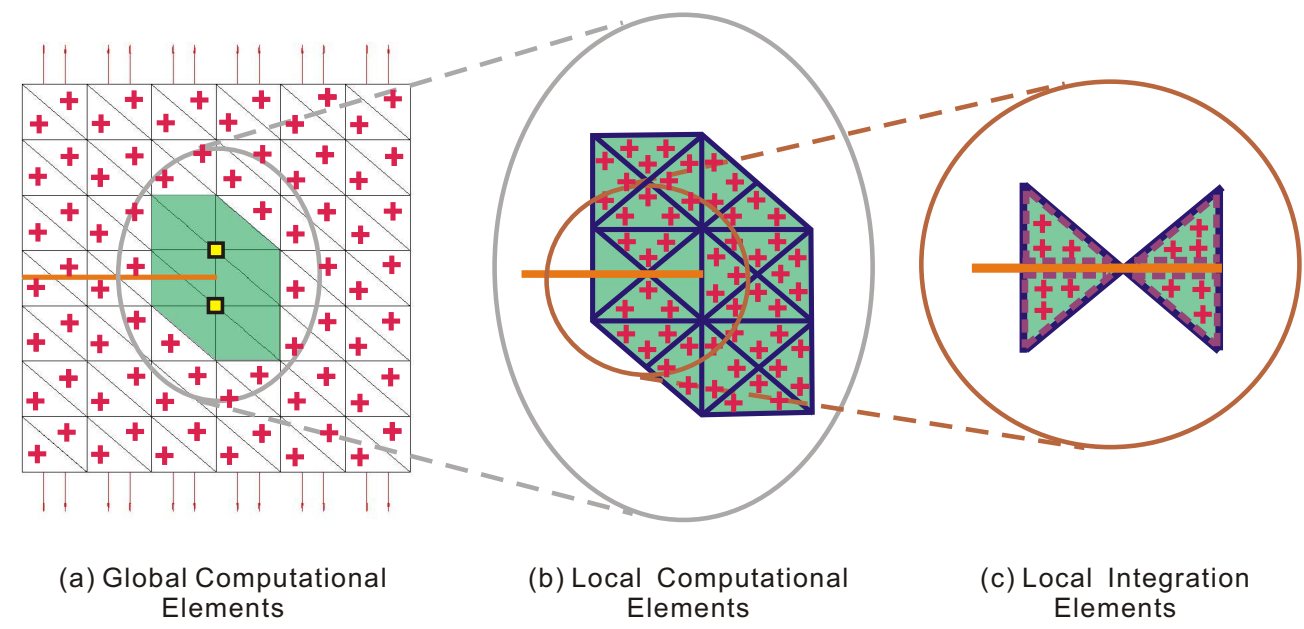

Figure 4: Numerical integration scheme in the global elements enriched with local solutions. Red crosses represent quadrature points. Elements without crosses use their descendants to define quadrature points. (a) Global computational elements and nodes enriched with global-local functions. (b) Local computational elements used for computation of global-local functions and numerical integration over global elements. (c) Integration elements used in elements cut by crack surface or enriched with singular functions. They are indicated with dashed lines in the figure.

\section{Numerical Examples}

In this section, we investigate the accuracy, robustness and computational efficiency of the proposed $G F E M^{\mathrm{gl}}$. The $G F E M^{\mathrm{gl}}$ solutions of three-dimensional fracture mechanics problems are compared with those available in the literature and with solutions provided by the $h p$-GFEM presented in [57, 58]. We also present results for the global-local FEM (GL-FEM)-the solution of the local problem defined in 
(6). Strictly speaking, problem (6) is a global-local generalized FEM since $\boldsymbol{u}_{L}$ is computed with the $h p$ GFEM and not a standard FEM. However, both global-local methods suffer from the same limitations, and it is reasonable to assume that the conclusions drawn here are also valid for the global-local FEM.

In all examples, coarse, uncracked, global meshes are used-no cracks are defined in the initial global problems.

A single local problem is defined for each crack in the domain. The local problem meshes are automatically constructed from the union of global clouds that intersect the crack fronts. Details are presented in Section A.1 of [18].

The accuracy of FFEM $^{\mathrm{gl}}$ solutions are evaluated in terms of the strain energy norm and stress intensity factor extracted using the Cut-off Function Method (CFM) [56, 69]. In order to quantify the error of the stress intensity factor (SIF) extracted along a crack front, we use a normalized discrete $L^{2}$-norm of the difference between the computed SIF and the reference solution defined by

$$
e^{r}\left(K_{i}\right):=\frac{\left\|e_{i}\right\|_{L^{2}}}{\left\|\hat{K}_{i}\right\|_{L^{2}}}=\frac{\sqrt{\sum_{j=1}^{N_{\text {ext }}}\left(K_{i}^{j}-\hat{K}_{i}^{j}\right)^{2}}}{\sqrt{\sum_{j=1}^{N_{\text {ext }}}\left(\hat{K}_{i}^{j}\right)^{2}}}
$$

where $N_{\text {ext }}$ is the number of extraction points along the crack front, $\hat{K}_{i}^{j}$ and $K_{i}^{j}$ are the reference and computed stress intensity factor values for mode $i$ at the crack front point $j$, respectively.

\subsection{Small Surface Crack}

As a first example to demonstrate the effectiveness of the proposed $G F E M^{\mathrm{gl}}$, we analyze a small halfpenny surface-breaking crack as illustrated in Figure 5. This problem has been analyzed by several researchers $[59,60,71]$ using the finite element method, and thus reliable reference solutions for the mode I stress intensity factor, $K_{I}$, along the crack front are available. The following geometrical and material parameters are adopted: In-plane dimensions $2 b=2.0,2 h=2.0$; domain thickness $t=1.0$; crack radius $r=0.2$; Young's modulus $E=1.0$, Poisson's ratio $v=0.25$. The domain is loaded by a unity bending moment $M$ as illustrated in Figure 5.

The global domain is discretized with a uniform coarse mesh of $6 \times(10 \times 11 \times 4)$ tetrahedral elements as shown in Figure 6. This is quite a coarse mesh with element sizes almost equal to the crack radius. The coarse global problem is solved to provide boundary conditions to the local problem. A local problem is created by extracting elements from the coarse global mesh around the surface crack. The elements intersecting the crack front are bisected until an acceptable level of mesh refinement is achieved. Figure 7(a) shows the local mesh. The ratio of element size to characteristic crack length $\left(L_{e} / r\right)$ along the crack front is 0.0295 . Discontinuous and singular analytical enrichment functions presented in [57] are automatically assigned to local nodes in order to model the crack. The von Mises stress in the local domain is shown in Figure 7(b). The local solution is next used as enrichment functions in the coarse global problem as illustrated in Figure 8. Cubic polynomial shape functions are used in both global and local problems.

As discussed in Section 3.2, we can use Dirichlet, Cauchy/Spring or Neumann boundary conditions at the local boundary $\partial \Omega_{L} \backslash\left(\partial \Omega_{L} \cap \partial \Omega_{G}\right)$. In fact, Dirichlet and Neumann boundary conditions are special cases of Cauchy boundary conditions, depending on the choice of the spring stiffness. Thus, we perform a sensitivity analysis to investigate the effect of the spring stiffness on the quality of the 


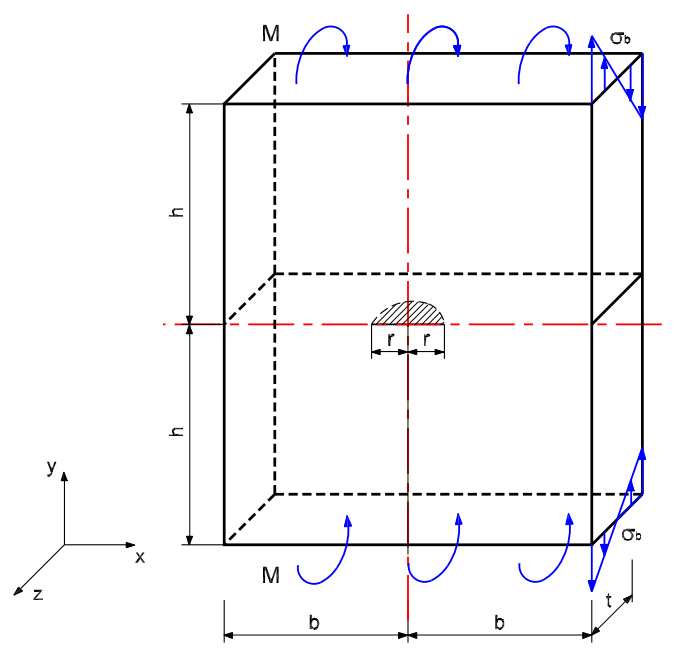

Figure 5: Domain with a small surface crack and loaded by a moment $M$. The resultant moment is applied using linearly varying tractions prescribed at faces of elements as shown in Figure 6.

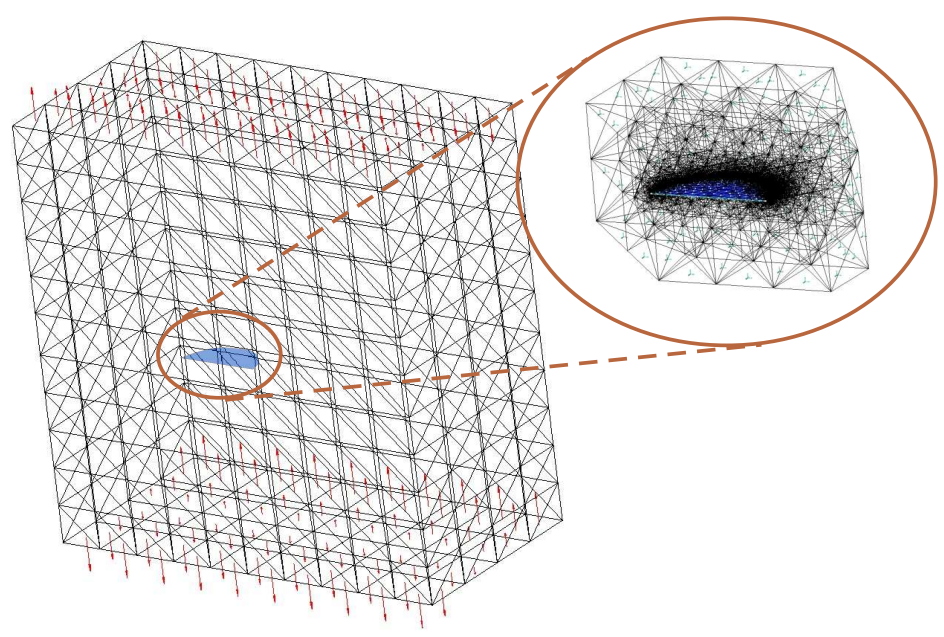

Figure 6: Coarse global mesh used to provide boundary conditions for local problem. The crack is not discretized in the global domain. The distributed tractions used to apply a bending moment at the top and bottom surfaces of the domain are also shown.

solution of the enriched global problem (11). Figure 9 plots the relative errors of the enriched global solution in energy norm for several spring stiffness values. The reference strain energy value is provided by the $h p-G F E M$ presented in [57]. The $h p-G F E M$ discretization is obtained by locally refining the 


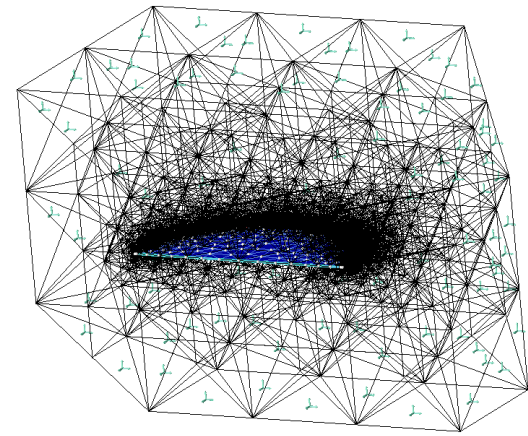

(a) $\mathrm{Hp}$-adapted local problem.

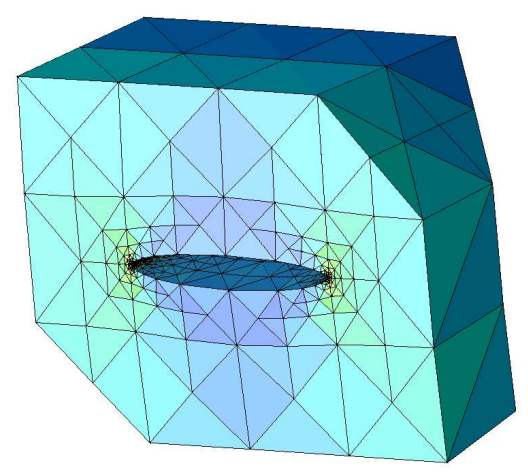

(b) Contour of von Mises stress of the local problem.

Figure 7: Local problem used to compute a global-local enrichment function.

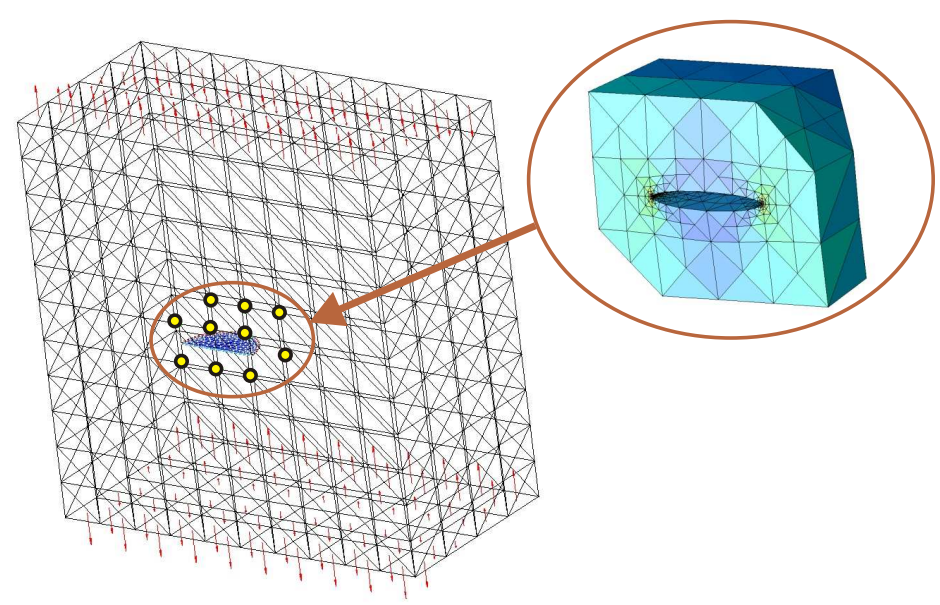

Figure 8: Hierarchical enrichment of the coarse global mesh with global-local functions. Yellow glyphs represent global nodes enriched with local solutions.

global mesh and enriching the global nodes with high-order shape functions as described in [57]. The crack is, in this case, discretized in the global domain. The relative errors of the $G F E M^{\mathrm{gl}}$ with Dirichlet and Neumann boundary conditions at $\partial \Omega_{L} \backslash\left(\partial \Omega_{L} \cap \partial \Omega_{G}\right)$ are also shown in the plot. It can be observed that the relative error of the spring boundary condition case is smaller than in the cases of Dirichlet and Neumann boundary conditions over the range of spring stiffness used in the plot. The figure shows a very smooth behavior and a low sensitivity of the global error with respect to the spring stiffness $\kappa$.

Selection of Spring Stiffness In this example, Neumann boundary conditions $(\kappa=0)$ can be used in the local problem since the solution of the uncracked global domain is exact and the tractions 


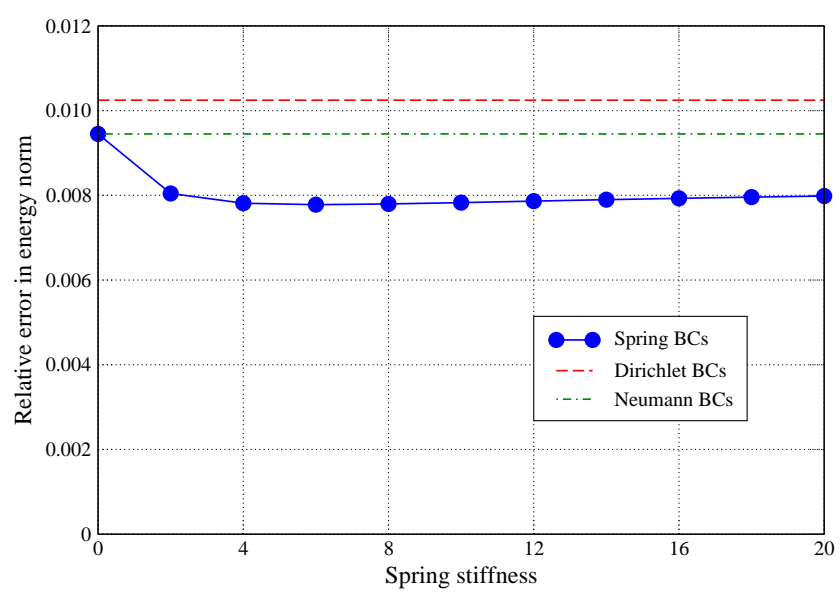

Figure 9: Sensitivity analysis to the stiffness of spring boundary conditions.

computed from it can equilibrate any local domain. However, this is not the case in general. Very small spring stiffness should also be avoided since this may be numerically equivalent to Neumann boundary conditions. Based on our numerical experience, the spring stiffness $\kappa$ is selected using the following expression

$$
\kappa=\frac{E}{\sqrt[n]{V_{0} J}}
$$

where $E$ is the Young's modulus, $n$ is the number of spacial dimensions of the problem, $V_{0}$ is the volume of the master element used (tetrahedrons in this example), and $J$ is the Jacobian of the global element across the local boundary where the spring boundary condition is imposed. The quantity $\sqrt[n]{V_{0} J}$ represents the characteristic length of the global finite element across the local boundary where the spring boundary condition is imposed. In this problem, the Jacobian and material properties of all global elements are constant and the spring stiffness given by (16) is $\kappa=8.7358$. From Figure 9, we can observe that much smaller values could also be used. This spring stiffness leads to a relative error in energy norm equal to 0.007807 , while for the Dirichlet boundary condition case the error is 0.010246 .

Quality of Extracted Stress Intensity factors Mode I stress intensity factor, $K_{I}$, extracted along the crack front is normalized using

$$
\bar{K}_{I}=\frac{K_{I}}{t_{y} \sqrt{\frac{\pi r}{Q}}}
$$

where $Q$ is equal to 2.464 for a circular crack, $t_{y}=3 M / b t^{2}$ is the maximum bending stress and $r$ is the radius of the crack. Dimensions $b$ and $t$ are indicated in Figure 5. The reference values for $\bar{K}_{I}$ are taken from Walters et al. [71] and used to compute $e^{r}\left(\bar{K}_{I}\right)$ in (15).

Figure 10 shows $\bar{K}_{I}$ computed with three methods-the GL-FEM, the GFEM ${ }^{\mathrm{gl}}$ and the $h p$-GFEM. The global-local FEM (GL-FEM) corresponds to SIF computed from the solution of the local problem shown in Figure 7 and subjected to spring boundary conditions provided by the initial global problem. 
The spring stiffness is given by (16). This approach provides a poor approximation of $\bar{K}_{I}$ along the crack front and the relative error $e^{r}\left(\bar{K}_{I}\right)$ is 0.18531 . The GFEM with global-local enrichment functions $\left(G F E M^{\mathrm{gl}}\right)$ corresponds to the case in which the local solution computed with the GL-FEM is used as enrichment function for the coarse global mesh shown in Figure 6 . The relative error $e^{r}\left(\bar{K}_{I}\right)$ of the FFEM $^{\mathrm{gl}} \mathrm{SIF}$ is 0.01233 , which is about 15 times smaller than the one obtained by the GL-FEM. We also show $h p$-GFEM results in the figure. The relative error $e^{r}\left(\bar{K}_{I}\right)$ of the $h p$-GFEM SIF is 0.00395 . While this result is quite accurate, the $h p$-GFEM requires refinement of the global mesh. The reference solution provided by Walters et al. [71] is also shown in the plot.

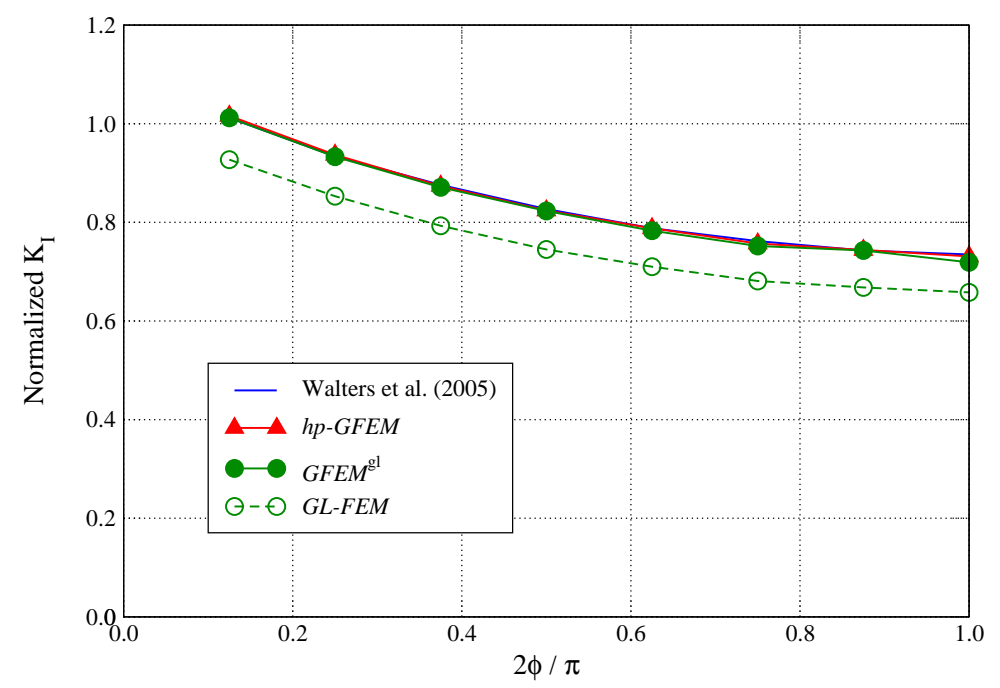

Figure 10: Normalized mode I stress intensity factor for the $G L-F E M$ and the $G F E M^{\mathrm{gl}}$ with spring boundary condition in local problems and the $h p$-GFEM. 


\subsection{Inclined Penny Shaped Crack}

The second problem is an inclined circular crack in a cube as illustrated Figure 11. The slope of the crack with respect to the global $y$-axis is $\gamma=\pi / 4$. A tensile traction of magnitude $\sigma$ is applied in the $y$-direction at the top and bottom surfaces of the domain. The following parameters are assumed in this problem: Cube dimension $2 L=4.0$; crack radius $a=1.0$; vertical traction $\sigma=1.0$; Young's modulus $E=1.0 ;$ Poisson's ratio $v=0.3$.

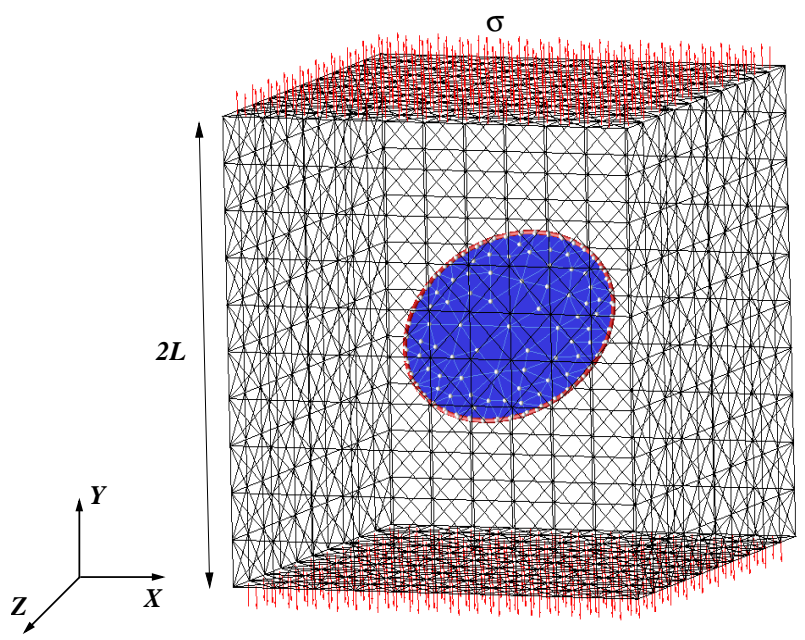

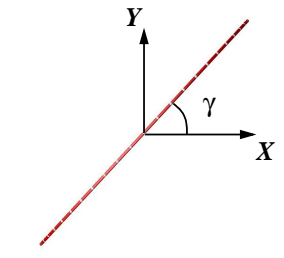

Right view

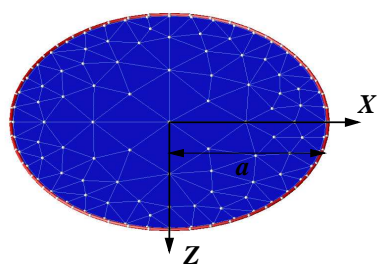

Top view

Figure 11: Inclined circular crack in a cube subjected to uniform tensile tractions. The crack is shown for illustration purposes only. It is not discretized in the initial global problem. The triangulation of the crack surface is also shown.

In this example, the stress intensity factors for all three modes are non-zero. We analyze the performance of the proposed $G F E M^{\mathrm{gl}}$ for this class of problems. The performance of the three types of boundary conditions applied at the local boundary $\partial \Omega_{L} \backslash\left(\partial \Omega_{L} \cap \partial \Omega_{G}\right)$ is also investigated.

The GFEM $^{\mathrm{gl}}$ solution is computed following the same steps described in the previous section. The global domain is discretized with a uniform coarse mesh of $6 \times(10 \times 10 \times 10)$ tetrahedral elements as shown in Figure 12. The crack is not discretized in the initial global domain. A single local problem is created along the circular crack front as shown in Figures 12 and 13. We can observe that the local domain does not contain the entire crack surface. The mesh and crack sizes were selected such that this would be the case. The mesh is locally refined around the crack front as shown in Figure 13(a). The ratio of element size to characteristic crack length $\left(L_{e} / a\right)$ along the crack front is 0.0280 . The von Mises stress in the local domain is shown in Figure 13(b). Figure 14 illustrates the enrichment of the coarse global mesh with the solution of the local problem. Cubic polynomial shape functions are used in both global and local problems.

In contrast with the problem of Section 4.1, the boundary of the local domain intersects the crack surface. While we could, of course, have used a larger local domain and avoid this situation, we are interested in the performance of the three types of boundary conditions applied at $\partial \Omega_{L} \backslash\left(\partial \Omega_{L} \cap \partial \Omega_{G}\right)$ under this situation. Note also that in this example $\partial \Omega_{L} \cap \partial \Omega_{G}=\emptyset$. Figure 15 shows the local 


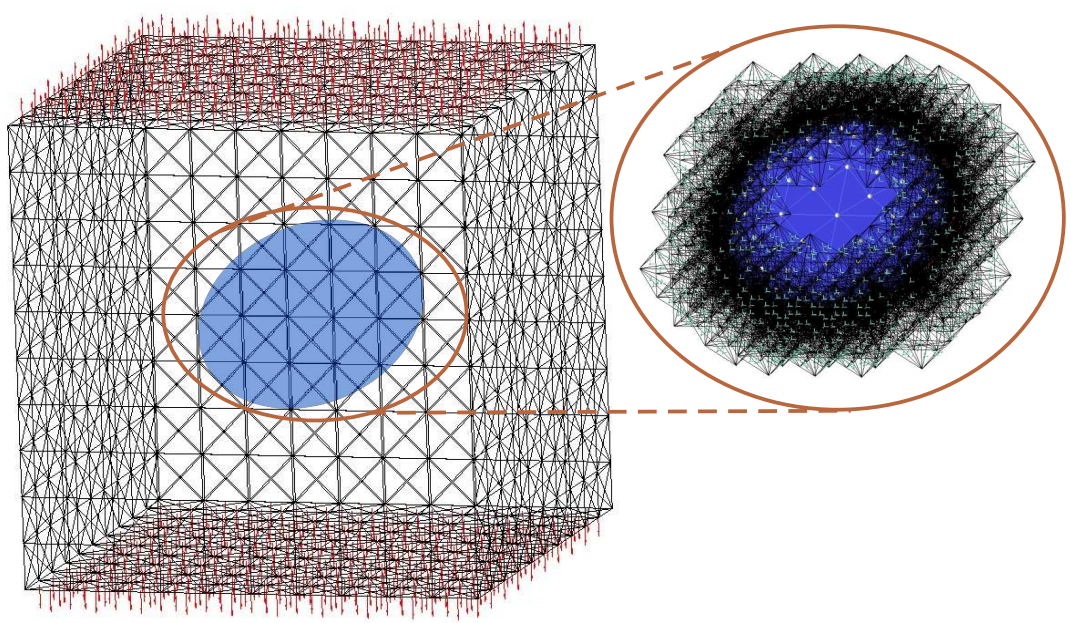

Figure 12: Coarse global mesh used to provide boundary conditions for the local problem. No crack is discretized in the global domain.

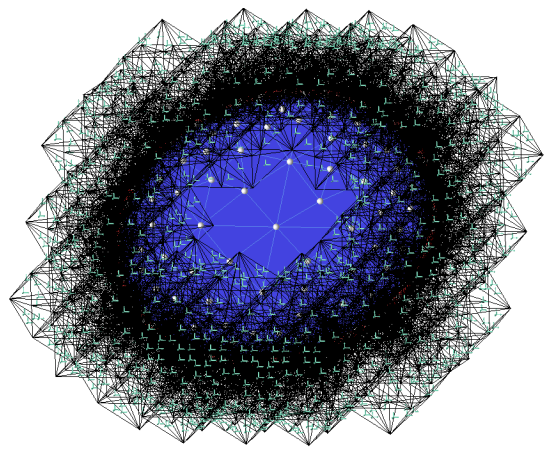

(a) $H p$-adapted local problem.

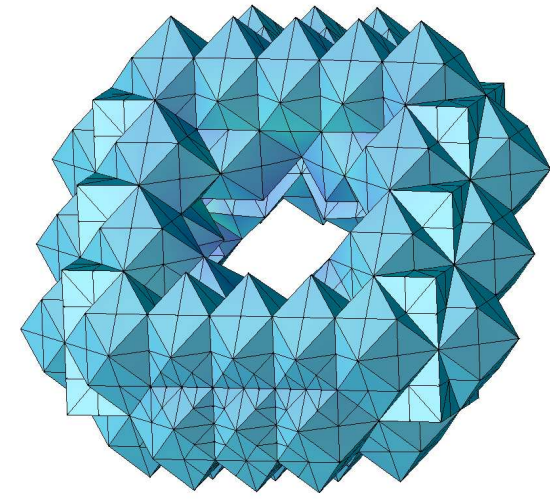

(b) Contour of von Mises stress of the local problem.

Figure 13: Local problem created along the circular crack front. The solution of the local problem is used as enrichment functions for the coarse global mesh.

deformed configurations for each type of boundary condition. We can observe in Figure 15(a) that the crack closes at the boundary of the local problem when Dirichlet boundary conditions are applied at $\partial \Omega_{L} \backslash\left(\partial \Omega_{L} \cap \partial \Omega_{G}\right)$. This is expected since the crack was not defined in the initial global problem and thus the Dirichlet boundary condition used at $\partial \Omega_{L} \backslash\left(\partial \Omega_{L} \cap \partial \Omega_{G}\right)$ is a continuous function. This behavior is not observed in Figures 15(b) and 15(c) which correspond to spring and Neumann boundary conditions, respectively. Since Neumann boundary conditions provided by the initial global problem are in general not equilibrated, spring boundary condition is the most robust option. A quantitative 


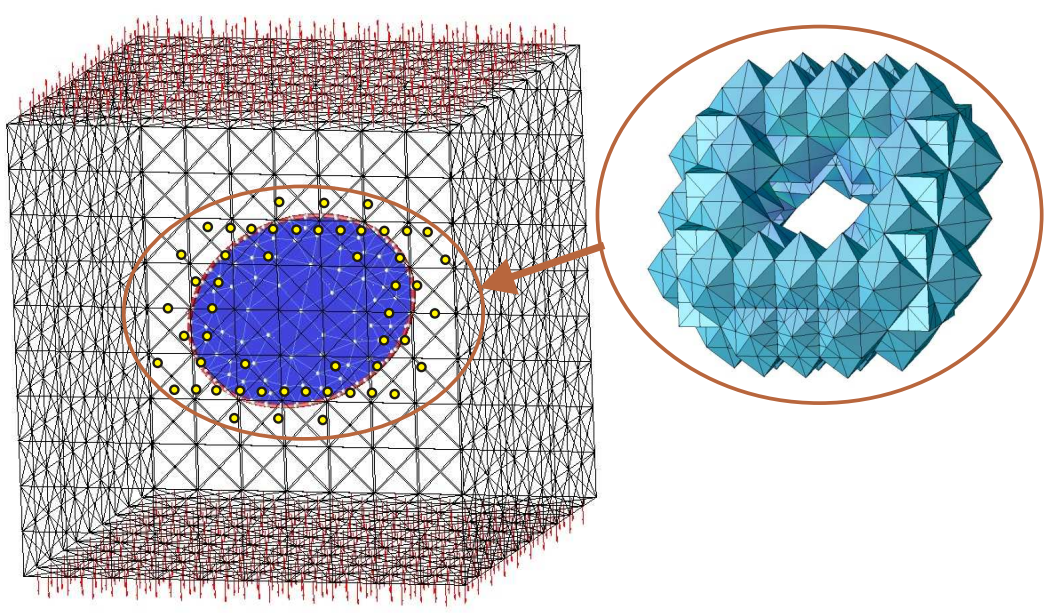

Figure 14: Enrichment of the global mesh with global-local functions. Yellow glyphs represent the global nodes enriched with these functions.

comparison among the three types of boundary conditions is presented below.

Selection of Spring Stiffness Figure 16 plots the relative error in energy norm of the enriched global solution for several spring stiffness values. The reference value for the strain energy is provided by using the $h p$-GFEM. Like in the problem analyzed in Section 4.1, the relative error of the spring boundary condition case is smaller than in the cases of Dirichlet and Neumann boundary conditions over a large range of spring stiffness values. The spring stiffness computed using (16) is $\kappa=7.2112$. While this is not the optimal value and much smaller values could also be used, it delivers more accurate results than Dirichlet and Neumann boundary conditions. We can also observe that in spite of the crack closing behavior caused by the Dirichlet boundary condition (Cf. Figure 15(a)), it is able to deliver accurate results.

Quality of Extracted Stress Intensity factors Figure 17 shows the mode I, II and III stress intensity factor distributions extracted along the crack front. The SIFs are extracted from solutions computed by three methods-the GL-FEM, GFEM ${ }^{\mathrm{gl}}$ and $h p$-GFEM. Spring boundary conditions with stiffness given by (16) are used in the global-local FEM. The local domain is shown in Figure 13. This GL-FEM solution is used as enrichment for the GFEM ${ }^{\mathrm{gl}}$. The SIFs extracted from the $h p$-GFEM solution are taken as reference values. The relative differences $e^{r}\left(K_{I}\right), e^{r}\left(K_{I I}\right)$ and $e^{r}\left(K_{I I I}\right)$ between the GFEM ${ }^{\mathrm{gl}}$ and $h p$-GFEM SIFs are 0.01420, 0.01748 and 0.02435, while those between the GL-FEM and $h p$-GFEM SIFs are $0.47515,0.43925$ and 0.33831 , respectively. The errors in all three mode SIFs computed with the $G F E M^{\mathrm{gl}}$ are one order of magnitude smaller than those with the GL-FEM. This, again, demonstrates the accuracy and robustness of the proposed $G F E M^{\mathrm{gl}}$. 


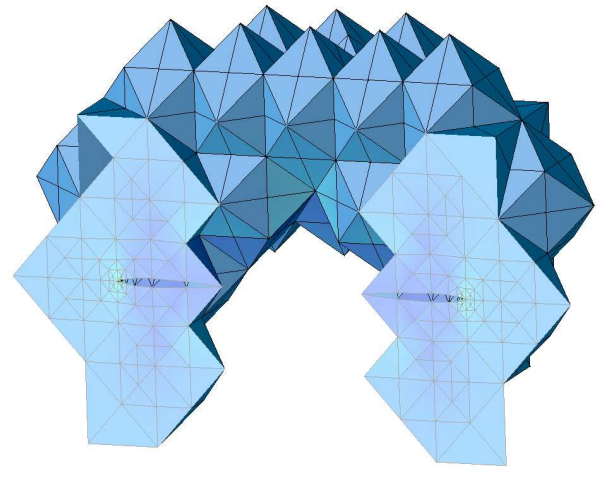

(a) Dirichlet boundary condition.

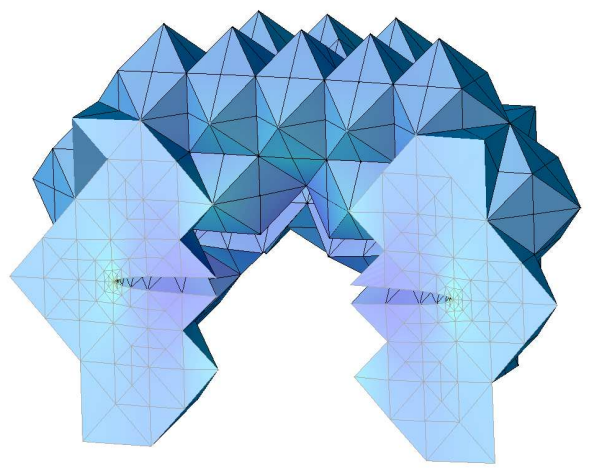

(b) Spring boundary condition.

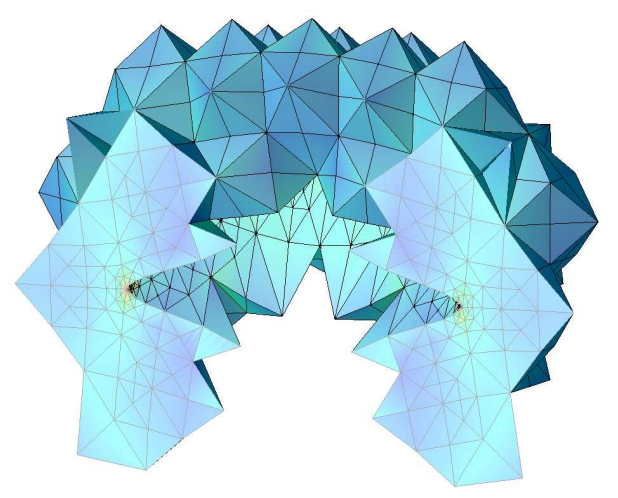

(c) Neumann boundary condition.

Figure 15: Section of local domain showing deformed shapes corresponding to three types of boundary conditions on $\partial \Omega_{L} \backslash\left(\partial \Omega_{L} \cap \partial \Omega_{G}\right)$. All the figures are drawn to the same scale. 


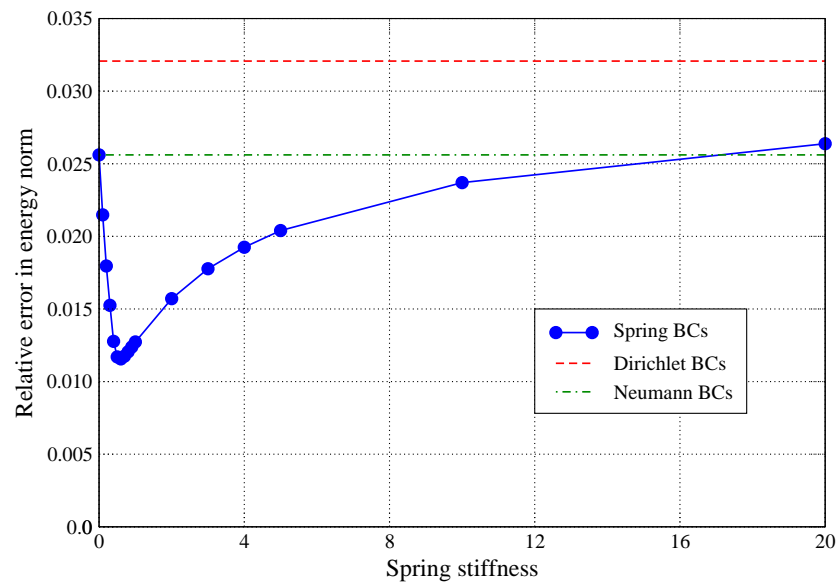

Figure 16: Sensitivity analysis to the stiffness of spring boundary conditions.

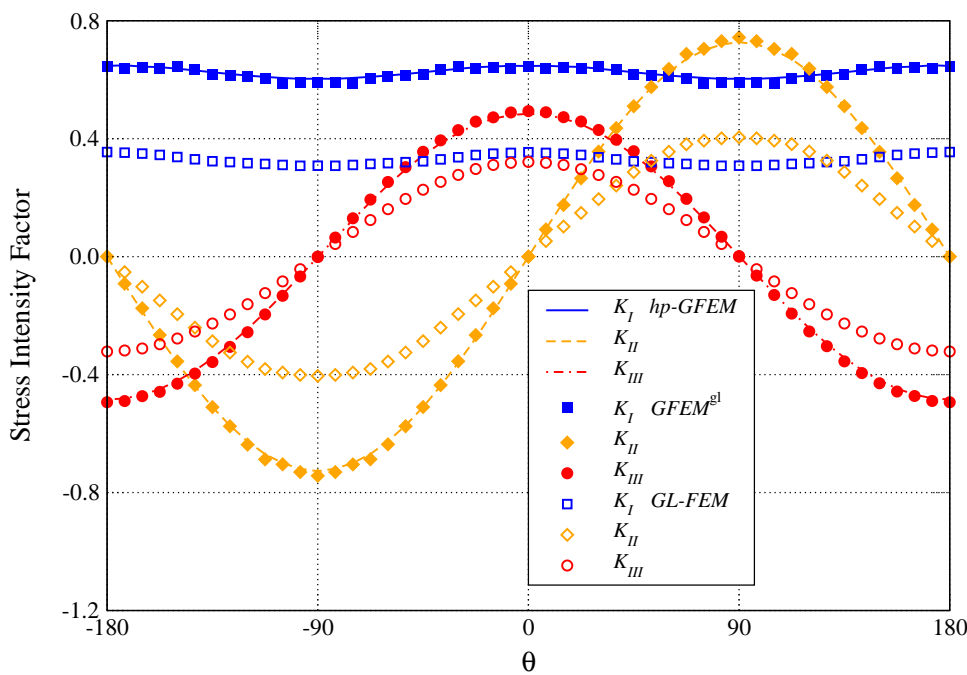

Figure 17: Stress intensity factors extracted from $G L-F E M, G F E M^{\mathrm{gl}}$ and $h p-G F E M$ solutions. Spring boundary conditions are used in the local problem. 


\subsection{Complex Domain with Multiple Crack Configurations}

In this section, we analyze the three-dimensional bracket shown in Figure 18 with the goal of demonstrating the computational efficiency of the proposed $G F E M^{\mathrm{gl}}$. The geometry of the domain and loads create several regions with stress singularities where cracks are likely to nucleate and grow. Three crack cases are considered as illustrated in the figure. In the proposed $G F E M^{\mathrm{gl}}$, the initial global problem needs to be solved only once and the same global coarse mesh can be used for any crack location. This feature of the method leads to substantial computational savings as demonstrated below. In contrast, the problem must be solved from scratch for each crack case when using, e.g., the finite element method. This type of analysis is frequently performed in the industry in order to find the critical crack location in a complex component [14]. The geometry and location of the cracks considered here are defined in Figure 21. The Young's modulus and Poisson's ratio used in this example are $E=10^{5}$ and $v=0.33$, respectively. The bracket is loaded by a unity pressure applied at the horizontal opening and it is fixed at the vertical openings.

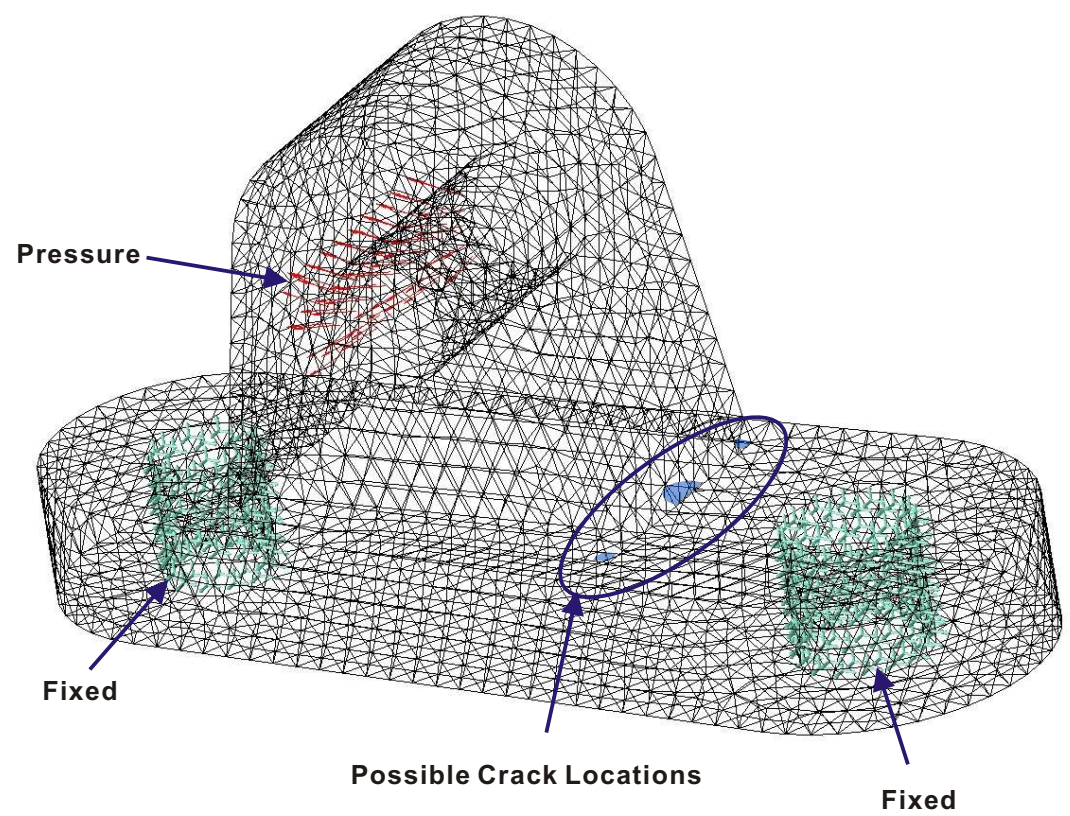

Figure 18: Boundary conditions and mesh for a three dimensional bracket. The three crack cases considered are shown in the figure but only one crack is analyzed at a time.

The coarse global problem is solved only once without any crack discretization and local problems are created around each crack as shown in Figure 19. The local meshes are refined around the crack fronts as in the previous sections. Spring boundary conditions provided by the global problem are used in all cases. The spring stiffness is, again, selected using (16). The von Mises stress distribution for the local problems are displayed in Figure 20. The coarse global mesh is enriched with one local solution at a time, as illustrated in Figure 21. The enriched global problems are then solved using the scheme discussed in Section 3.4. The GFEM $^{\mathrm{gl}}$ solution for each crack location considered is shown in Figure 
22.

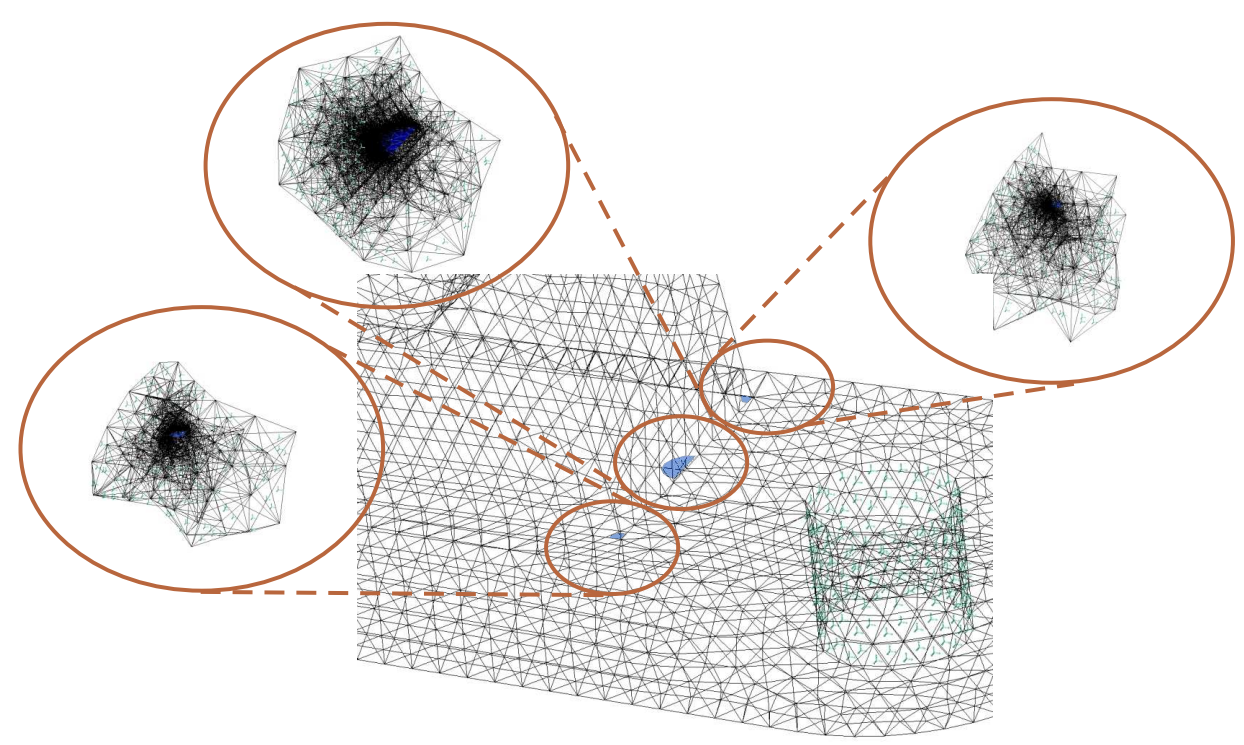

Figure 19: The solution of the initial global problem provides boundary conditions for local problems created around each crack surface. No crack is discretized in the global domain and thus it needs to be solved only once.

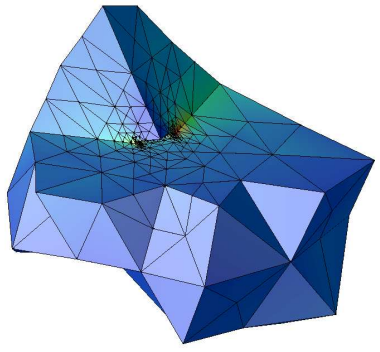

(a) Crack 1 (Quarter circle).

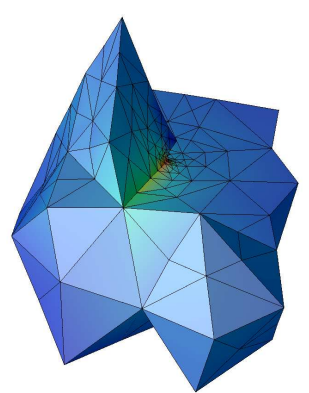

(b) Crack 2 (Quarter circle).

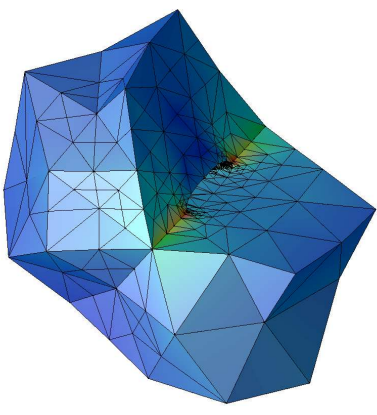

(c) Crack 3 (Half circle).

Figure 20: The von Mises stress distribution for the local problems. Spring boundary conditions provided by the same initial global solution are used in all cases. 


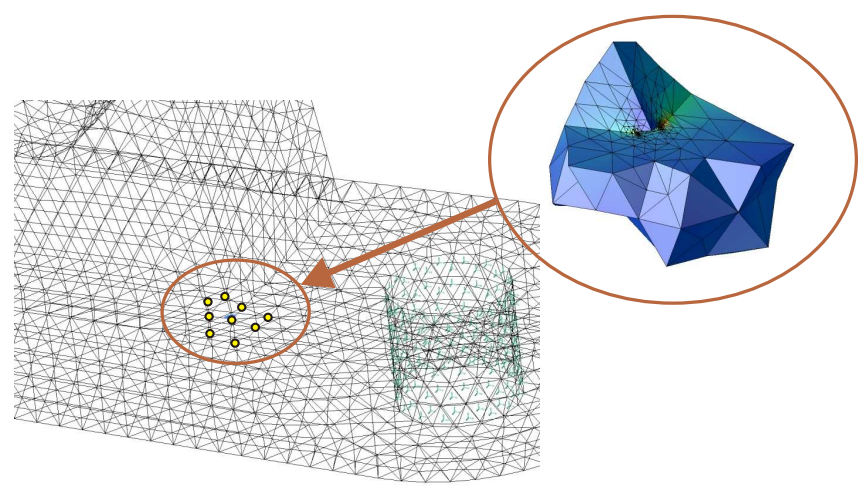

(a)

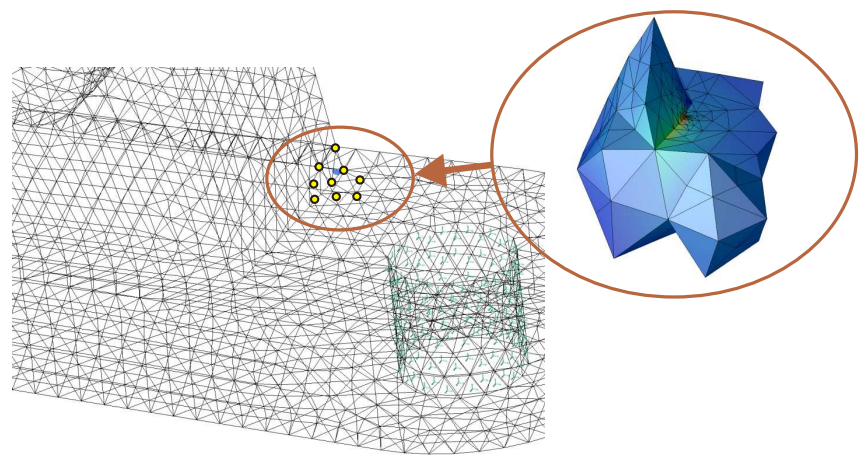

(b)

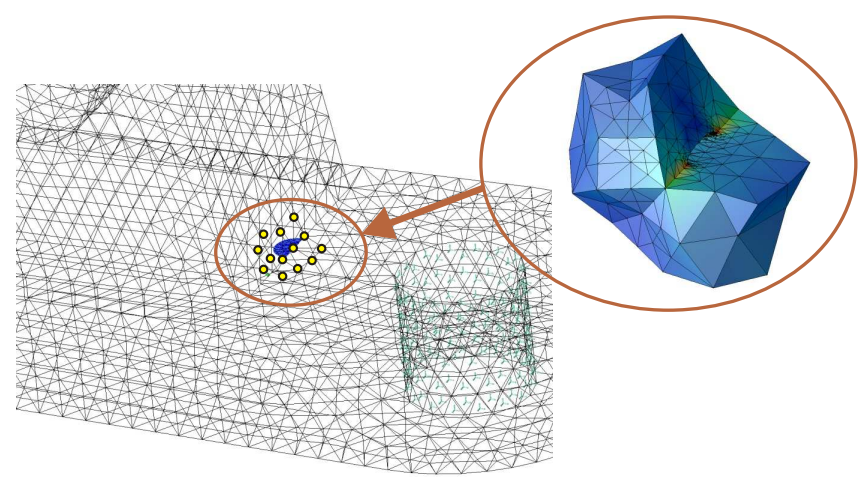

(c)

Figure 21: Enrichment of the coarse global mesh with the local solution of each crack case considered. The geometry of each crack is as follows: (a) Crack 1 (Quarter circle): Radius $r=4$, center $=(80,50,50)$; $(\phi=0, r=4)=(80,50,46) ;(\phi=\pi / 2, r=4)=(76,50,50) ;(b)$ Crack 2 (Quarter circle): Radius $r=4$, center $=(80,50,-50) ;(\phi=0, r=4)=(76,50,-50) ;(\phi=\pi / 2, r=4)=(80,50,-46) ;(\mathrm{c})$ Crack 3 (Half circle): Radius $r=8$, center $=(80,50,0) ;(\phi=0, r=8)=(80,50,-8) ;(\phi=\pi / 2, r=8)=(72,50,0)(\phi=\pi, r=8)=(80,50,8)$. Where $(\phi, r)$ are polar coordinates along the crack front and $(\phi, r)=(X, Y, Z)$ means the Cartesian coordinates of point $(\phi, r)$ located along the crack front.

[GFEMgl' two 'scale - July 1, 2009] 


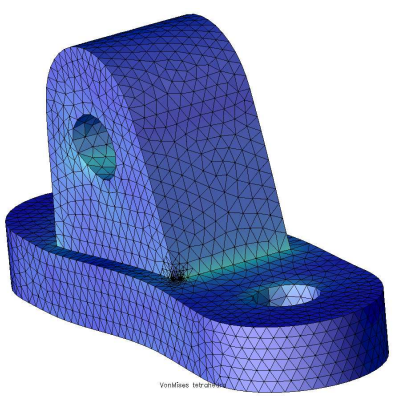

(a) Crack 1

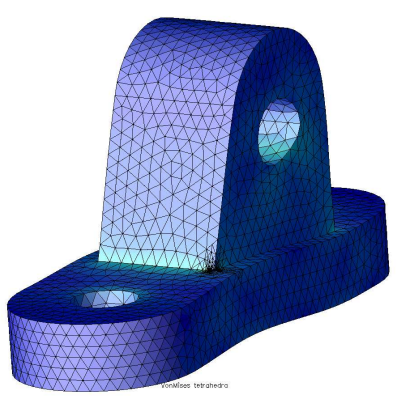

(b) Crack 2 .

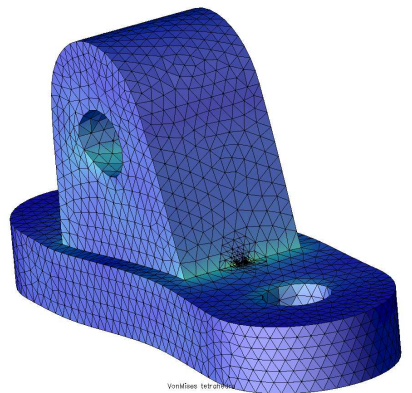

(c) Crack 3 .

Figure 22: $G F E M^{\mathrm{gl}}$ solution for each crack location considered.

Computational Performance To evaluate the computational efficiency of the proposed FFEM $^{\mathrm{gl}}$, we compare its computational cost with that of the hp-GFEM. In the latter, like in the classical FEM, the problem must be solved from scratch for each crack configuration. This leads to high computational costs when a large number of crack configurations must be analyzed as is the case of crack growth simulations or multiple site damage (MSD) analysis. In contrast, in the $G F E M^{\mathrm{gl}}$, the factorized stiffness matrix of the uncracked global problem can be used to compute the solution of enriched global problems at a low computational cost.

Tables I and II list the CPU time required to solve the three crack cases using the hp-GFEM and the $G F E M^{\mathrm{gl}}$, respectively. The number of degrees of freedom used by each method is also listed. Several observations can be made from the result in the tables.

First, the size of the enriched global problem in the GFEM ${ }^{\mathrm{gl}}$ does not depend on that of the local problem. Furthermore, only a small number of degrees of freedom are added to the enriched global problem: 27, 27 and 39 for the first, second and third crack case, respectively. In contrast, the crack discretization and mesh refinement required by the $h p-G F E M$ increase the size of the global problem substantially.

Second, the cost to compute the enriched global solutions corresponds to only between 4 and $6 \%$ of the CPU time spent in the initial global problem $(298.7 s)$. As a results, the total CPU time for the GFEM $^{\mathrm{gl}}$ is much smaller than that required by the $h p$-GFEM. The difference in performance between the two methods grows with the number of crack configurations considered. This is clearly demonstrated in Figure 23, which shows the total CPU time versus the number of crack configurations for the $G F E M^{\mathrm{gl}}$ and $h p-G F E M$. If the solution of the uncracked global problem is available from the design phase of the component, the total CPU time for the $G F E M^{\mathrm{gl}}$ is $152.7 \mathrm{~s}$ since it involves only the solution of the local and enriched global problems. Otherwise, the CPU time for the GFEM ${ }^{\mathrm{gl}}$ is $451.4 s$. In either case, the $G F E M^{\mathrm{gl}}$ is considerably more efficient than the $h p$-GFEM.

Quality of Extracted Stress Intensity factors Figure 24 shows mode I, II and III stress intensity factors extracted along the front of Crack 3. Both the $h p-G F E M$ and GFEM $^{\mathrm{gl}}$ solutions are shown. The SIFs extracted from the GFEM ${ }^{\mathrm{gl}}$ solution are in good agreement with those from the hp-GFEM solution. The relative differences $e^{r}\left(K_{I}\right), e^{r}\left(K_{I I}\right)$ and $e^{r}\left(K_{I I I}\right)$ between the GFEM ${ }^{\mathrm{gl}}$ and $h p$-GFEM SIFs are $0.02454,0.04902$ and 0.03275 , respectively. This demonstrates that the $G_{F E M^{\mathrm{gl}}}$ can deliver 
Table I: CPU time spent on the factorization of the stiffness matrix of each crack case using the $h p$-GFEM.

\begin{tabular}{c|c|c|c}
\hline Crack & Number of degrees of freedom & CPU time (sec) & Strain energy \\
\hline 1 & 139,098 & 346.7 & 2.3638 \\
2 & 136,698 & 335.6 & 2.3634 \\
3 & 157,626 & 459.9 & 2.3752 \\
\hline \multicolumn{2}{c}{ Total } & $1,142.2$ & \\
\hline
\end{tabular}

Table II: CPU time spent on the factorization of the initial and local problems and on the solution of the enriched global problems. If the solution of the initial (uncracked) global problem is not available, the total CPU time of the $G F E M^{\mathrm{gl}}$ to solve the three crack cases is 451.4 instead of 152.7 .

\begin{tabular}{c|c|c|c|c|c|c|c|c}
\hline \multirow{2}{*}{ Crack ID } & \multicolumn{2}{|c|}{ Number of degrees of freedom } & \multicolumn{4}{c|}{ CPU time (sec) } & \multirow{2}{*}{} \\
\cline { 2 - 8 } & Initial & Local & Enriched & Initial & Local & Enriched & Total & Strain energy \\
\hline 1 & & 23,268 & 115,497 & & 24.1 & 12.6 & 36.6 & 2.3633 \\
2 & 115,470 & 21,108 & 115,497 & 298.7 & 17.3 & 12.6 & 29.9 & 2.3631 \\
3 & & 39,426 & 115,509 & & 69.3 & 16.9 & 86.2 & 2.3735 \\
\hline \multicolumn{3}{|c|}{ Total } & 298.7 & 110.7 & 42.0 & 152.7 & \\
\hline
\end{tabular}

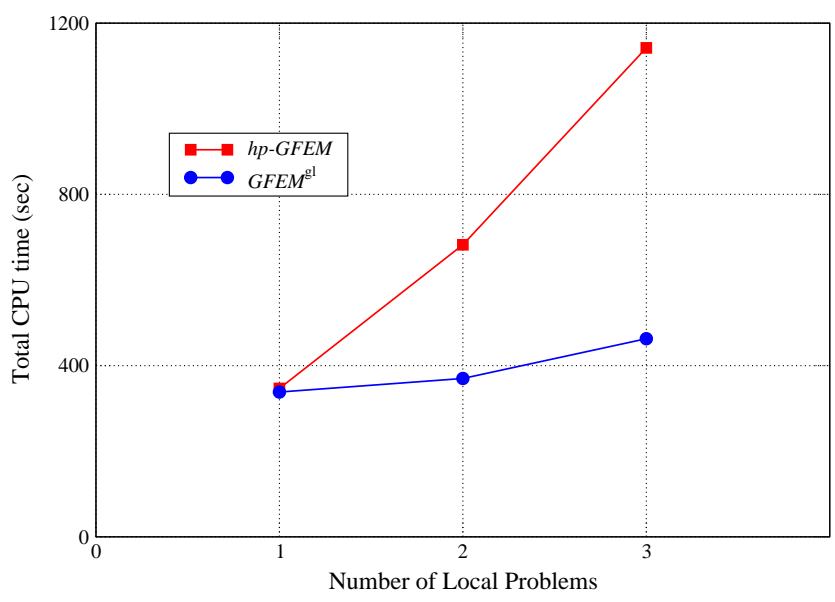

Figure 23: Total CPU time required for a different number of crack configurations in the $G F E M^{\mathrm{gl}}$ and $h p-G F E M$ analyses. The cost of solving the uncracked global problem was included in the the CPU time of the GFEM ${ }^{\mathrm{gl}}$.

accurate stress intensity factors at a lower computational cost than the $h p-G F E M$.

[GFEMgl'two`scale - July 1, 2009] 


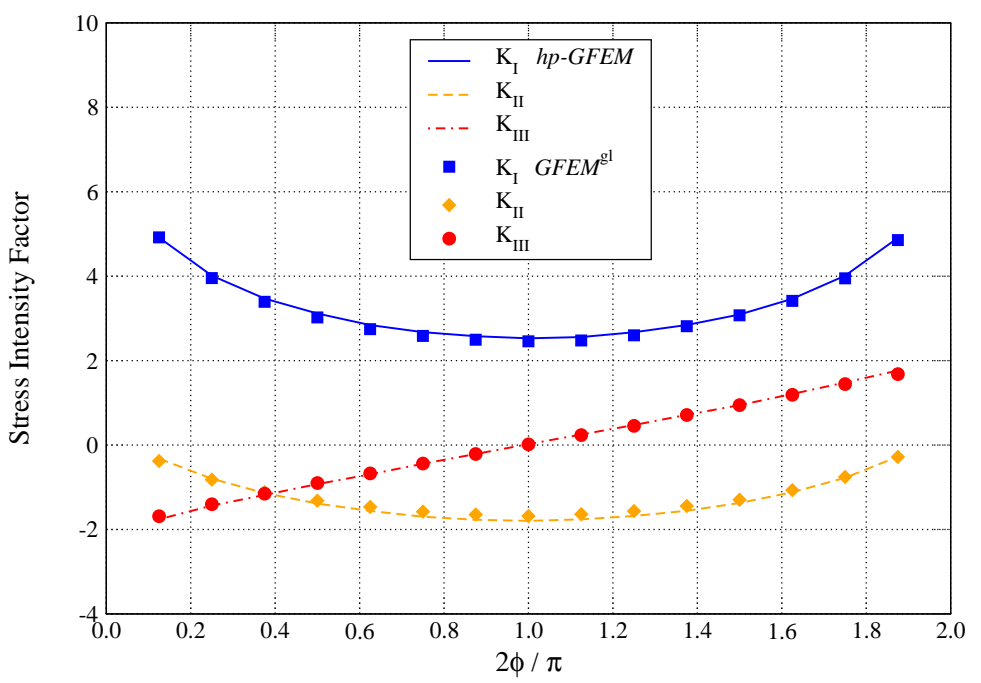

Figure 24: Mode I, II and III stress intensity factors extracted from $h p-G F E M$ and GFEM $^{\mathrm{gl}}$ solutions for Crack 3. Spring boundary conditions are used in the local problem.

\section{Conclusions}

The examples presented in Section 4 show that stress intensity factors extracted from the proposed $G F E M^{\mathrm{gl}}$ solutions are up to one order of magnitude more accurate than those extracted from the local solutions. The latter case is equivalent to the global-local FEM ( GL-FEM), broadly used in the industry. The numerical examples also demonstrate that the accuracy of the $G F E M^{\mathrm{gl}}$ is comparable with that of the $h p-G F E M$ proposed in [57] while not requiring the refinement of global meshes. This enables, for example, the use of meshes available from the design phase of a structure to perform fracture mechanics analyses.

The computational cost in terms of CPU time of the proposed GFEM $^{\mathrm{gl}}$ is comparable with that of the GL-FEM. The only additional cost of the former is the solution of the enriched global problem. This, as demonstrated in Section 4.3, is small when compared with the solution of the initial global problem.

In the proposed $G F E M^{\mathrm{gl}}$, the initial global problem needs to be solved only once and the same global coarse mesh can be used for any crack configuration. This feature of the method leads to substantial computational savings when several crack configurations are considered in the same structure. In contrast, the global problem must be solved from scratch for each crack configuration when using, e.g., the finite element method. Similar conclusions are expected in the case of crack propagation simulations. We are currently investigating this case.

The $h p-G F E M$ is as accurate and computationally efficient as the standard FEM due to the use of singular crack front enrichment functions. Thus, it is reasonable to assume that the above conclusions regarding accuracy and computational efficiency of the $G F E M^{\mathrm{gl}}$ and $h p-G F E M$ also applies to the standard FEM.

Another contribution of this paper is a study of the accuracy of the FEEM $^{\mathrm{gl}}$ when Dirichlet,

[GFEMgl'two'scale - July 1, 2009] 
Cauchy/spring or Neumann boundary conditions provided by the initial global problem are used at the local boundary $\partial \Omega_{L} \backslash\left(\partial \Omega_{L} \cap \partial \Omega_{G}\right)$. Our numerical experiments show that any value of the spring stiffness comparable to, or larger than the stiffness of the body is acceptable and provides global-local enrichment functions with good approximation properties. This type of boundary condition leads, in general, to more accurate enriched global solutions than Dirichlet boundary conditions while being more robust than Neumann boundary conditions.

Acknowledgments: The authors gratefully acknowledge the contributions of the Midwest Structural Sciences Center (MSSC) at the University of Illinois at Urbana-Champaign. The Center is supported by the U.S. Air Force Research Laboratory Air Vehicles Directorate under contract number FA865006-2-3620. The support from the U.S. Air Force Research Laboratory Air Vehicles Directorate under contract number USAF-0060-50-0001 is also gratefully acknowledged.

\section{REFERENCES}

[1] A.M. Aragon, C.A. Duarte, and Ph.H. Geubelle. Generalized finite element enrichment functions for discontinuous gradient fields. International Journal for Numerical Methods in Engineering, 2009. Submitted for publication. 1

[2] I. Babuška and B. Andersson. The splitting method as a tool for multiple damage analysis. SIAM Journal on Scientific Computing, 26:1114-1145, 2005. 2

[3] I. Babuška and J.M. Melenk. The partition of unity finite element method. Technical Report BN-1185, Inst. for Phys. Sc. and Tech., University of Maryland, June 1995. 1, 3

[4] I. Babuška and J.M. Melenk. The partition of unity finite element method. International Journal for Numerical Methods in Engineering, 40:727-758, 1997. 1, 3

[5] I. Babuška, G. Caloz, and J.E. Osborn. Special finite element methods for a class of second order elliptic problems with rough coefficients. SIAM Journal on Numerical Analysis, 31(4):745-981, 1994. 3

[6] I. Babuška, F. Ihlenburg, E. Paik, and S. Sauter. A generalized finite element method for solving the Helmholtz equation in two dimensions with minimal pollution. Computer Methods in Applied Mechanics and Engineering, 128(3-4):325-360, 1995. 1

[7] J. Bellec and J. Dolbow. A note on enrichment functions for modelling crack nucleation. Communications in Numerical Methods in Engineering, 19:921-932, 2003. 2

[8] T. Belytschko and T. Black. Elastic crack growth in finite elements with minimal remeshing. International Journal for Numerical Methods in Engineering, 45:601-620, 1999. 1, 3

[9] T. Belytschko, J. Fish, and A. Bayliss. The spectral overlay on finite elements for problems with high gradients. Computer Methods in Applied Mechanics and Engineering, 81:71-89, 1990. 2

[10] T. Belytschko, S. Loehnert, and J.-H. Song. Multiscale aggregating discontinuities: A method for circumventing loss of material stability. International Journal for Numerical Methods in Engineering, 73:869-894, 2007. 2 
[11] P. Bochev and R.B. Lehoucq. On the finite element solution of the pure Neumann problem. SIAM Review, 47(1):50-66, 2005. 7

[12] D.L. Chopp and N. Sukumar. Fatigue crack propagation of multiple coplanar cracks with the coupled extended finite element/fast marching method. International Journal of Engineering Science, 41:845-869, 2003. 4

[13] M. Cloirec, N. Moës, G. Marckmann, and P. Cartraud. Two-scale analysis of cracks using the extended finite element method. In D.R.J. Owen, E. Onate, and B. Suarez, editors, VIII International Conference on Computational Plasticity Fundamentals and Application COMPLAS 2005, pages 483-485, Barcelona, Spain, 2005. CIMNE. 2

[14] A.Th. Diamantoudis and G.N. Labeas. Stress intensity factors of semi-elliptical surface cracks in pressure vessels by global-local finite element methodology. Engineering Fracture Mechanics, 72:1299-1312, 2005. 22

[15] C.A. Duarte. The hp Cloud Method. PhD dissertation, The University of Texas at Austin, December 1996. Austin, TX, USA. 3

[16] C.A. Duarte and I. Babuška. Mesh-independent directional p-enrichment using the generalized finite element method. International Journal for Numerical Methods in Engineering, 55(12): 1477-1492, 2002. http://dx.doi.org/10.1002/nme.557. 1

[17] C.A. Duarte and I. Babuška. A global-local approach for the construction of enrichment functions for the generalized fem and its application to propagating three-dimensional cracks. In V.M.A. Leitão, C.J.S. Alves, and C.A. Duarte, editors, ECCOMAS Thematic Conference on Meshless Methods, Lisbon, Portugal, 11-14 July 2005. 8 pages. 2, 4, 5

[18] C.A. Duarte and D.-J. Kim. Analysis and applications of a generalized finite element method with global-local enrichment functions. Computer Methods in Applied Mechanics and Engineering, 197(6-8):487-504, 2008. http://dx.doi.org/10.1016/j.cma.2007.08.017. 2, 4, 5, 7, 9, 10, 11, 12

[19] C.A. Duarte, I. Babuška, and J.T. Oden. Generalized finite element methods for three dimensional structural mechanics problems. Computers and Structures, 77:215-232, 2000. 1, 3, 4

[20] C.A. Duarte, O.N. Hamzeh, T.J. Liszka, and W.W. Tworzydlo. A generalized finite element method for the simulation of three-dimensional dynamic crack propagation. Computer Methods in Applied Mechanics and Engineering, 190(15-17):2227-2262, 2001. http://dx.doi.org/10.1016/S0045-7825(00)00233-4. 1, 4

[21] C.A. Duarte, D.-J. Kim, and I. Babuška. Chapter: A global-local approach for the construction of enrichment functions for the generalized fem and its application to three-dimensional cracks. In V.M.A. Leitão, C.J.S. Alves, and C.A. Duarte, editors, Advances in Meshfree Techniques, volume 5 of Computational Methods in Applied Sciences, The Netherlands, 2007. Springer. ISBN 978-1-4020-6094-6. 2, 4, 5

[22] C.A.M. Duarte and J.T. Oden. Hp clouds-A meshless method to solve boundary-value problems. Technical Report 95-05, TICAM, The University of Texas at Austin, May 1995. 3

[23] C.A.M. Duarte and J.T. Oden. Hp clouds-An $h p$ meshless method. Numerical Methods for Partial Differential Equations, 12:673-705, 1996. 3

[GFEMgl'two`scale - July 1, 2009] 
[24] C.A.M. Duarte and J.T. Oden. An $h p$ adaptive method using clouds. Computer Methods in Applied Mechanics and Engineering, 139:237-262, 1996. 3

[25] M. Duflot and S. Bordas. XFEM and mesh adaptation: A marriage of convenience. In Eighth World Congress on Computational Mechanics, Venice, Italy, July 2008. 1

[26] A. Düster. High order finite elements for three-dimensional, thin-walled nonlinear continua. Shaker Verlag, Aachen, Germany, 2002. 10

[27] A. Düster, A. Niggl, and E. Rank. Applying the hp-d version of the fem to locally enhance dimensionally reduced models. Computer Methods in Applied Mechanics and Engineering, 196: 3524-3533, 2007. 2, 10

[28] R. Fan and J. Fish. The rs-method for material failure simulations. International Journal for Numerical Methods in Engineering, 73(11):1607-1623, 2008. doi: 10.1002/nme.2134. 3

[29] C.A. Felippa. Introduction to finite element methods., 2004. Course Notes. Department of Aerospace Engineeing Sciences, University of Colorado at Boulder. Available at http://www.colorado.edu/engineering/Aerospace/CAS/courses.d/IFEM.d. 10

[30] J. Fish. The s-version of the finite element method. Computers and Structures, 43:539-547, 1992. 2,3

[31] J. Fish and R. Guttal. The s-version of finite element method for laminated composites. International Journal for Numerical Methods in Engineering, 39:3641-3662, 1996.

[32] J. Fish and A. Nath. Adaptive and hierarchical modelling of fatigue crack propagation. International Journal for Numerical Methods in Engineering, 36:2825-2836, 1993. 2

[33] S. Ghosh, K. Lee, and P. Raghavan. A multi-level computational model for multi-scale analysis in composite and porous materials. International Journal of Solids and Structures, 38:2335-2385, 2001. 2

[34] S. Ghosh, J. Bai, and P. Raghavan. Concurrent multi-level model for damage evolution in microstructurally debonding composites. Mechanics of Materials, 39(3):241-266, 2007. 2

[35] A. Gravouil, N. Moës, and T. Belytschko. Non-planar 3d crack growth by the extended finite element and level sets - Part II: Level set update. International Journal for Numerical Methods in Engineering, 53(11):2569-2586, 2002. 1, 4

[36] P.-A. Guidault, O. Allix, L. Champaney, and C. Cornuault. A multiscale extended finite element method for crack propagation. Computer Methods in Applied Mechanics and Engineering, 197: 381-399, 2008. 2, 10

[37] I. Hirai, B.P. Wang, and W.D. Pilkey. An efficient zooming method for finite element analysis. International Journal for Numerical Methods in Engineering, 20:1671-1683, 1984. 10

[38] I. Hirai, Y. Uchiyama, Y. Mizuta, and W.D. Pilkey. An exact zooming method. Finite Elements in Analysis and Design, 1:61-69, 1985. 10

[39] T.Y. Hou and X.-H. Wu. A multiscale finite element method for elliptic problems in composite materials and porous media. Journal of Computational Physics, 134:169-189, 1997. 9

[GFEMgl'two`scale - July 1, 2009] 
[40] D.-J. Kim, C.A. Duarte, and J.P. Pereira. Analysis of interacting cracks using the generalized finite element method with global-local enrichment functions. ASME Journal of Applied Mechanics, 75(5), 2008. 051107 (12 pages) http://dx.doi.org/10.1115/1.2936240. 2

[41] R. Krause and E. Rank. Multiscale computations with a combination of the h- and p-versions of the finite-element method. Computer Methods in Applied Mechanics and Engineering, 192: 3959-3983, 2003. 2, 10

[42] S.-H. Lee, J.-H. Song, Y.-C. Yoon, G. Zi, and T. Belytschko. Combined extended and superimposed finite element method for cracks. International Journal for Numerical Methods in Engineering, 59(1119-1136), 2004. DOI: 10.1002/nme.908. 2, 10

[43] W.K. Liu and C. McVeigh. Predictive multiscale theory for design of heterogenous materials. Computational Mechanics, 42(2):147-170, 2008. DOI: 10.1007/s00466-007-0176-8. 2

[44] S. Loehnert and T. Belytschko. A multiscale projection method for macro/microcrack simulations. International Journal for Numerical Methods in Engineering, 71(12):1466-1482, 2007. 2

[45] K.M. Mao and C.T. Sun. A refined global-local finite element analysis method. International Journal for Numerical Methods in Engineering, 32:29-43, 1991. 2

[46] C. McVeigh and W.K. Liu. Linking microstructure and properties through a predictive multiresolution continuum. Computer Methods in Applied Mechanics and Engineering, 197(4142):3268-3290, 2008. 2

[47] C. McVeigh, F. Vernerey, W.K. Liu, and L.C. Brinson. Multiresolution analysis for material design. Computer Methods in Applied Mechanics and Engineering, 195(37-40):5053-5076, 2006. 2

[48] J.M. Melenk. On Generalized Finite Element Methods. PhD thesis, The University of Maryland, 1995. 1

[49] J.M. Melenk and I. Babuška. The partition of unity finite element method: Basic theory and applications. Computer Methods in Applied Mechanics and Engineering, 139:289-314, 1996. 3

[50] N. Moës, J. Dolbow, and T. Belytschko. A finite element method for crack growth without remeshing. International Journal for Numerical Methods in Engineering, 46:131-150, 1999. 1,3

[51] N. Moës, A. Gravouil, and T. Belytschko. Non-planar 3D crack growth by the extended finite element and level sets - Part I: Mechanical model. International Journal for Numerical Methods in Engineering, 53(11):2549-2568, 2002. 1, 4

[52] J.T. Oden, C.A. Duarte, and O.C. Zienkiewicz. A new cloud-based $h p$ finite element method. Computer Methods in Applied Mechanics and Engineering, 153:117-126, 1998. 1, 3

[53] P. O'Hara, C.A. Duarte, T. Eason, and D.-J. Kim. Generalized finite element analysis of three-dimensional heat transfer problems exhibiting sharp thermal gradients. Computer Methods in Applied Mechanics and Engineering, 198(21-26):1857-1871, 2009. http://dx.doi.org/10.1016/j.cma.2008.12.024. 2

[GFEMgl'two 'scale - July 1, 2009] 
[54] C. Oskay and J. Fish. On calibration and validation of eigendeformation-based multiscale models for failure analysis of heterogeneous systems. Computational Mechanics, 42(2):181-195, 2008. 3

[55] K. Park, J.P. Pereira, C.A. Duarte, and G.H. Paulino. Integration of singular enrichment functions in the generalized/extended finite element method for three-dimensional problems. International Journal for Numerical Methods in Engineering, 78(10):1220-1257, 2009. http://dx.doi.org/10.1002/nme.2530. 10

[56] J.P. Pereira and C.A. Duarte. Extraction of stress intensity factors from generalized finite element solutions. Engineering Analysis with Boundary Elements, 29:397-413, 2005. 12

[57] J.P. Pereira, C.A. Duarte, D. Guoy, and X. Jiao. Hp-Generalized FEM and crack surface representation for non-planar 3-D cracks. International Journal for Numerical Methods in Engineering, 77(5):601-633, 2009. http://dx.doi.org/10.1002/nme.2419. 1, 2, 4, 5, 6, 10, 11, $12,13,14,27$

[58] J.P. Pereira, C.A. Duarte, X. Jiao, and D. Guoy. Generalized finite element method enrichment functions for curved singularities in 3D fracture mechanics problems. Computational Mechanics, 44(1):73-92, 2009. http://dx.doi.org/10.1007/s00466-008-0356-1. 2, 4, 6, 11

[59] J.C. Raju, I.S. Newman Jr. Stress-intensity factors for a wide range of semi-elliptical surface cracks in finite-thickness plates. Engineering Fracture Mechanics, 11:817-829, 1979. 12

[60] J.C. Raju, I.S. Newman Jr. Three dimensional finite-element analysis of finite-thickness fracture specimens. Report TN D-8414, NASA - Langley Research Center, Hampton, VA, May 1977. pp. 1-40. 12

[61] J. Rannou, A. Gravouil, and M.C Baietto-Dubourg. A local multigrid X-FEM strategy for 3-D crack propagation. International Journal for Numerical Methods in Engineering, 2009. DOI: 10.1002/nme.2427. 2

[62] A. Simone, C.A. Duarte, and E. van der Giessen. A generalized finite element method for polycrystals with discontinuous grain boundaries. International Journal for Numerical Methods in Engineering, 67(8):1122-1145, 2006. http://dx.doi.org/10.1002/nme.1658. 1

[63] T. Strouboulis, K. Copps, and I. Babuška. The generalized finite element method. Computer Methods in Applied Mechanics and Engineering, 190:4081-4193, 2001. 1, 3, 9

[64] T. Strouboulis, L. Zhang, and I. Babuška. Generalized finite element method using mesh-based handbooks: Application to problems in domains with many voids. Computer Methods in Applied Mechanics and Engineering, 192:3109-3161, 2003.

[65] T. Strouboulis, L. Zhang, and I. Babuška. p-version of the generalized FEM using meshbased handbooks with applications to multiscale problems. International Journal for Numerical Methods in Engineering, 60:1639-1672, 2004. 9

[66] N. Sukumar, N. Moës, B. Moran, and T. Belytschko. Extended finite element method for threedimensional crack modelling. International Journal for Numerical Methods in Engineering, 48 (11):1549-1570, 2000. 1, 4

[GFEMgl'two`scale - July 1, 2009] 
[67] N. Sukumar, D.L. Chopp, and B. Moran. Extended finite element method and fast marching method for three-dimensional fatigue crack propagation. Engineering Fracture Mechanics, 70: 29-48, 2003. 1,4

[68] B. Szabo and I. Babuška. Finite Element Analysis. John Wiley and Sons, New York, 1991. 7

[69] B. A. Szabo and I. Babuška. Computation of the amplitude of stress singular terms for cracks and reentrant corners. In T.A. Cruse, editor, Fracture Mechanics: Nineteenth Symposium, ASTM STP 969, pages 101-124, Southwest Research Institute, San Antonio, TX, 1988. 12

[70] I. Tsukanov and V. Shapiro. Adaptive multiresolution refinement with distance fields. International Journal for Numerical Methods in Engineering, 72:1355-1386, 2007. DOI: 10.1002/nme.2087. 2

[71] M. C. Walters, G. H. Paulino, and R. H. Dodds Jr. Stress-intensity factors for surface cracks in functionally graded materials under mode-I thermomechanical loading. International Journal of Solids and Structures, 41:1081-1118, 2004. 12, 15, 16

[72] J.D. Whitcomb. Iterative global/local finite element analysis. Computers and Structures, 40: 1027-1031, 1991. 10

[73] E. Wyart, D. Coulon, M. Duflot, T. Pardoen, J.-F. Remacle, and F. Lani. A substructured FEshell/XFE-3D method for crack analysis in thin-walled structures. International Journal for Numerical Methods in Engineering, 72:757-779, 2007. 10

[74] E. Wyart, M. Duflot, D. Coulon, P. Martiny, T. Pardoen, J.-F. Remacle, and F. Lani. Substructuring FE-XFE approaches applied to three-dimensional crack propagation. Journal of Computational and Applied Mathematics, 2007. doi: 10.1016/j.cam.2006.03.066. 10 